\title{
Genomic variation in 3,010 diverse accessions of Asian cultivated rice
}

Wensheng Wang ${ }^{1,17}$, Ramil Mauleon ${ }^{2,17}$, Zhiqiang Hü1,3,17, Dmytro Chebotarov²,17, Shuaishuai Tai ${ }^{4,17}$, Zhichao Wu ${ }^{1,5,17}$, Min Li ${ }^{6,7,17}$, Tianqing Zheng ${ }^{1,17}$, Roven Rommel Fuentes ${ }^{2,17}$, Fan Zhang ${ }^{1,17}$, Locedie Mansueto ${ }^{2,17}$, Dario Copetti ${ }^{2,8,17}$, Millicent Sanciangco ${ }^{2}$, Kevin Christian Palis ${ }^{2}$, Jianlong Xu ${ }^{1,5,6}$, Chen Sun ${ }^{3}$, Binying Fu ${ }^{1,6}$, Hongliang Zhang ${ }^{9}$, Yongming Gao ${ }^{1,6}$, Xiuqin Zhao ${ }^{1}$, Fei Shen ${ }^{9}$, Xiao Cui ${ }^{3}$, Hong Yu ${ }^{10}$, Zichao Li ${ }^{9}$, Miaolin Chen ${ }^{3}$, Jeffrey Detras ${ }^{2}$, Yongli Zhou ${ }^{1,6}$, Xinyuan Zhang ${ }^{5}$, Yue Zhao ${ }^{3}$, Dave Kudrna ${ }^{8}$, Chunchao Wang ${ }^{1}$, Rui Li ${ }^{3}$, Ben $\mathrm{Jia}^{3}$, Jinyuan $\mathrm{Lu}^{3}$, Xianchang $\mathrm{He}^{3}$, Zhaotong Dong ${ }^{3}$, Jiabao Xu${ }^{4}$, Yanhong $\mathrm{Li}^{4}$, Miao Wang ${ }^{4}$, Jianxin Shi ${ }^{3}$, Jing $\mathrm{Li}^{3}$, Dabing Zhang ${ }^{3}$, Seunghee Lee ${ }^{8}$, Wushu Hu${ }^{4}$, Alexander Poliakov ${ }^{11}$, Inna Dubchak ${ }^{11,12}$, Victor Jun Ulat ${ }^{2}$, Frances Nikki Borja ${ }^{2}$, John Robert Mendoza ${ }^{13}$, Jauhar $\mathrm{Ali}^{2}$, Jing $\mathrm{Li}^{3}$, Qiang $\mathrm{GaO}^{4}$, Yongchao Niu ${ }^{4}$, Zhen Yue ${ }^{4}$, Ma. Elizabeth B. Naredo ${ }^{2}$, Jayson Talag ${ }^{8}$, Xueqiang Wang ${ }^{9}$, Jinjie Li ${ }^{9}$, Xiaodong Fang ${ }^{4}$, Ye Yin ${ }^{4}$, Jean-Christophe Glaszmann ${ }^{14,15}$, Jianwei Zhang ${ }^{8}$, Jiayang Li ${ }^{1,10}$, Ruaraidh Sackville Hamilton ${ }^{2}$, Rod A. Wing $2,8 *$, Jue Ruan ${ }^{5 *}$, Gengyun Zhang ${ }^{4,6 *}$, Chaochun Wei ${ }^{3,16 *}$, Nickolai Alexandrov ${ }^{2 *}$, Kenneth L. McNally ${ }^{2 *}$, Zhikang Li ${ }^{1,6 *} \&$ Hei Leung $^{2}$

Here we analyse genetic variation, population structure and diversity among 3,010 diverse Asian cultivated rice (Oryza sativa L.) genomes from the 3,000 Rice Genomes Project. Our results are consistent with the five major groups previously recognized, but also suggest several unreported subpopulations that correlate with geographic location. We identified 29 million single nucleotide polymorphisms, 2.4 million small indels and over 90,000 structural variations that contribute to within- and between-population variation. Using pan-genome analyses, we identified more than 10,000 novel full-length protein-coding genes and a high number of presence-absence variations. The complex patterns of introgression observed in domestication genes are consistent with multiple independent rice domestication events. The public availability of data from the 3,000 Rice Genomes Project provides a resource for rice genomics research and breeding.

Asian cultivated rice is grown worldwide and comprises the staple food for half of the global population. It is envisaged that by the year $2035^{1}$ feeding this growing population will necessitate that an additional 112 million metric tons of rice be produced on a smaller area of land, using less water and under more fluctuating climatic conditions, which will require that future rice cultivars be higher yielding and resilient to multiple abiotic and biotic stresses. The foundation of the continued improvement of rice cultivars is the rich genetic diversity within domesticated populations and wild relatives ${ }^{2-4}$. For over 2,000 years, two major types of O. sativa-O. sativa Xian group (here referred to as Xian/Indica (XI) and also known as 籼, Hsien or Indica) and O. sativa Geng Group (here referred to as Geng/Japonica (GJ) and also known as 粳, Keng or Japonica) - have historically been recognized ${ }^{5-7}$. Varied degrees of post-reproductive barriers exist between XI and GJ rice accessions $^{8}$; this differentiation between XI and GJ rice types and the presence of different varietal groups are well-documented at isozyme and DNA levels ${ }^{6,9}$. Two other distinct groups have also been recognized using molecular markers ${ }^{10}$; one of these encompasses the Aus, Boro and Rayada ecotypes from Bangladesh and India (which we term the circum-Aus group (cA)) and the other comprises the famous Basmati and Sadri aromatic varieties (which we term the circum-Basmati group (cB)).

Approximately 780,000 rice accessions are available in gene banks worldwide ${ }^{11}$. To enable the more efficient use of these accessions in future rice improvement, the Chinese Academy of Agricultural Sciences, BGI-Shenzhen and International Rice Research Institute sequenced over 3,000 rice genomes ( $3 K-R G$ ) as part of the 3,000 Rice Genomes Project ${ }^{12}$.

Here we present analyses of genetic variation in the $3 \mathrm{~K}-\mathrm{RG}$ that focus on important aspects of $O$. sativa diversity, single nucleotide polymorphisms (SNPs) and structural variation (deletions, duplications, inversions and translocations). We also construct a species pangenome consisting of 'core' genes that are present in all individuals and 'distributed' (variable, accessory or dispensable) genes that are absent in some individuals ${ }^{13,14}$. The gene presence-absence variations (PAVs) represent another component of species genetic diversity. Our analyses provide new perspectives on rice intra-species diversity and evolutionary history.

\section{Genome mapping, size and SNP variation}

Baseline genome sequencing, analyses, and accession information and metadata for the 3,024 rice genomes are summarized in Supplementary Data 1 and Supplementary Notes. Fourteen accessions were excluded from further analyses after quality control. The remaining 3,010 genomes had an average mapping coverage of $92 \%$ (74.6-98.7\%) (Supplementary Data 2 Table 1), when aligned to the O. sativa cv. Nipponbare IRGSP 1.0 reference genome ${ }^{15}$ (hereafter referred to as 'Nipponbare RefSeq'). The estimated size of the genome was

\footnotetext{
${ }^{1}$ Institute of Crop Sciences, Chinese Academy of Agricultural Sciences, Beijing, China. ${ }^{2}$ International Rice Research Institute, Manila, Philippines. ${ }^{3}$ School of Life Sciences and Biotechnology, Shanghai Jiao Tong University, Shanghai, China. ${ }^{4}$ BGI Genomics, BGI-Shenzhen, Shenzhen, China. ${ }^{5}$ Agricultural Genomics Institute, Chinese Academy of Agricultural Sciences, Shenzhen, China. ${ }^{6}$ Shenzhen Institute for Innovative Breeding, Chinese Academy of Agricultural Sciences, Shenzhen, China. ${ }^{7}$ Anhui Agricultural University, Hefei, China. ${ }^{8}$ Arizona Genomics Institute, School of Plant Sciences, University of Arizona, Tucson, AZ, USA. ${ }^{9}$ China Agricultural University, Beijing, China. ${ }^{10}$ Institute of Genetics and Developmental Biology, Chinese Academy of Sciences, Beijing, China. ${ }^{11}$ DOE Joint Genome Institute, Walnut Creek, CA, USA. ${ }^{12}$ Lawrence Berkeley National Laboratory, Berkeley, CA, USA. ${ }^{13}$ Advanced Science and Technology Institute, Department of Science and Technology, Quezon City, Philippines. ${ }^{14}$ UMR AGAP, CIRAD, Montpellier, France. ${ }^{15}$ UMR AGAP, Université de Montpellier, Montpellier, France. ${ }^{16}$ Shanghai Center for Bioinformation Technology, Shanghai, China. ${ }^{17}$ These authors contributed equally: Wensheng Wang, Ramil Mauleon, Zhiqiang Hu, Dmytro Chebotarov, Shuaishuai Tai, Zhichao Wu, Min Li, Tianqing Zheng, Roven Rommel Fuentes, Fan Zhang, Locedie Mansueto, Dario Copetti. *e-mail: lizhikang@caas.cn; k.mcnally@irri.org; ccwei@sjtu.edu.cn; ruanjue@caas.cn; nalexandrov@inariag.com; zhanggengyun@genomics.cn; rwing@ag.arizona.edu
} 


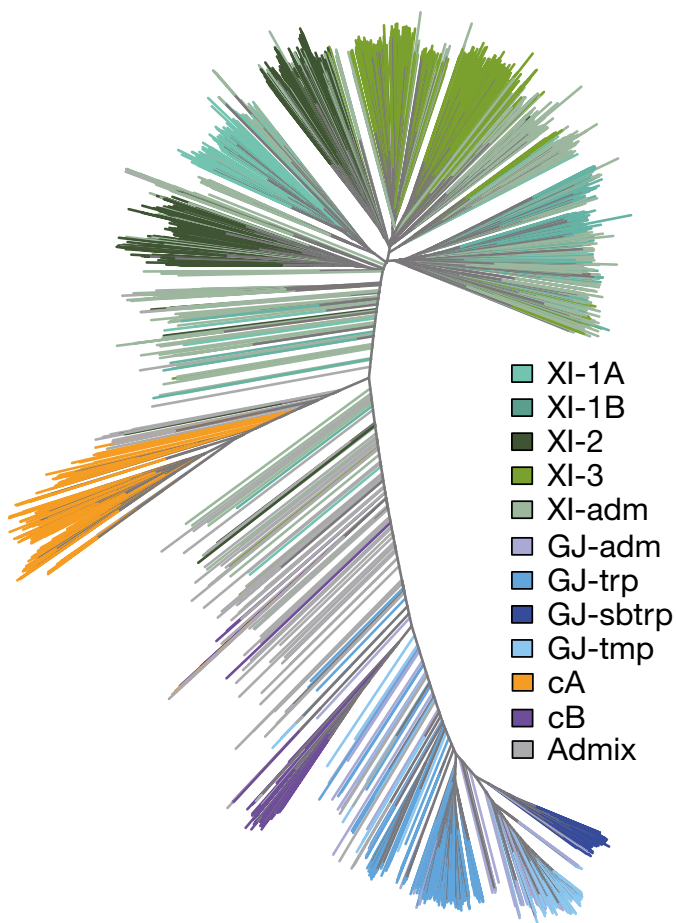

Fig. 1 | Unweighted neighbour-joining tree based on 3,010 samples and computed on a simple matching distance matrix for filtered SNPs. Samples are coloured by their assignment to $k=9$ subpopulations from ADMIXTURE ${ }^{46}$.

$375.1 \pm 20.9 \mathrm{Mb}$, with $42.5 \pm 0.4 \%$ guanine-cytosine content and 35.6 $\pm 3.7 \%$ repetitive sequence content (Supplementary Data 3 Table 1).

We identified over 29 million SNPs-27 million of which are biallelic-and found high concordance $(>96 \%)$ with previous reports (Supplementary Notes) ${ }^{16,17}$. Filtering reduced this to a 'base SNP' set of approximately 17 million SNPs, which captured $>99.9 \%$ of all SNPs with minor allele frequencies (MAF) $>0.25 \%$ (Extended Data Fig. 1). Half (56\%) of non-transposable element (NTE) genes and the majority (91\%) of transposable element (TE)-related genes have high-effect SNPs (Supplementary Data 2 Tables 2-4). NTE genes contained about 1.44 million moderate-to-high effect, and about 1.5 million low-effect, SNPs, which gave a ratio of 0.95 for moderate-to-high:low SNPs. For small indels, insertions affected $28 \%$ of NTE- and $50 \%$ of TE-related genes: deletions affected $41 \%$ of NTE- and $70 \%$ of TE-related genes. A typical genome in a major varietal group contains approximately 2 million (XI and cA), 0.3-0.8 million (GJ; depending on the subpopulation) or about 1.2 million (cB) SNPs (Supplementary Data 2 Table 5). The SNPs of a typical genome were classified as 7.9\% moderate-to-high effect and $5.1 \%$ low effect.

\section{Population structure and diversity}

The $3 \mathrm{~K}-\mathrm{RG}$ accessions were classified into nine subpopulations (Fig. 1 and Extended Data Fig. 2a-d), most of which could be connected to geographic origins (Supplementary Data 1). There were four XI clusters (XI-1A from East Asia, XI-1B of modern varieties of diverse origins, XI-2 from South Asia and XI-3 from Southeast Asia); three GJ clusters (primarily East Asian temperate (named GJ-tmp), Southeast Asian subtropical (named GJ-sbtrp) and Southeast Asian tropical (named GJ-trp)); and single groups for the mostly South Asian cA and $c B$ accessions. Accessions with admixture components $<0.65$ within $\mathrm{XI}$ and GJ were classified as 'XI-adm' and 'GJ-adm', respectively, and accessions that fell between major groups were classified as admixed (Extended Data Fig. 2a).

Distinct allele frequency profiles for SNPs of MAF $>10 \%$ occurred for the nine subpopulations with deviations from the neutral model reflecting different adaptations and demographic events (Extended Data Fig. 3a). Larger numbers of 'private' alleles were found in $\mathrm{cA}$ and $\mathrm{cB}$ than in other subpopulations (Extended Data Fig. 2e). Comparatively, XI subpopulations have smaller numbers of private alleles, probably owing to ongoing gene flow from natural hybridization and breeding. Doubleton sharing patterns within and between subpopulations showed the same trend (Extended Data Fig. 2f).

Linkage disequilibrium decay rates for combined subpopulations were higher in XI than GJ, with little variation between the two GJ subpopulations, as previously reported ${ }^{7,16,18}$. However, for the nine subpopulations, linkage disequilibrium decay between XI subpopulations varied more markedly, with XI-2 and XI-3 exhibiting faster linkage disequilibrium decay than XI-1A and XI-1B (Extended Data Fig. 3b). Furthermore, linkage disequilibrium decay correlates strongly with nucleotide diversity $(\pi)$ among the nine subpopulations $\left(R^{2}=0.93\right.$, $P$ value $\left.=2.5 \times 10^{-5}\right)($ Extended Data Fig. 3 c $)$.

Nucleotide diversity computation identified many regions of low genetic diversity that contained small numbers of genes under selective constraints (Extended Data Fig. 3d). Sh4 ${ }^{19}$, which controls nonshattering, showed an accordant profile of diversity reduction across all subpopulations (Fig. 2a) that indicates much longer selection, when compared to $q S H 1^{20}$. At the semi-dwarf gene $s d 1^{21}$ locus, a narrow region of reduced diversity occurred in all major groups, which is a similar pattern to that observed for $q S H 1$. However, higher diversity in the surrounding $100-\mathrm{kb}$ regions occurred in the $\mathrm{cA}, \mathrm{cB}$ and XI groups, whereas the GJ groups had extended regions of reduced diversity, which reflects the breeding history associated with the 'green revolution ${ }^{22}$. Different patterns of diversity reduction were observed at other important loci. The $W x^{23}$ locus that affects amylose content and stickiness on cooking, the Badh2.1 ${ }^{24}$ locus that affects aroma and their surrounding regions are highly diverse in the $\mathrm{XI}, \mathrm{cA}$ and $\mathrm{cB}$ groups, which indicates complex histories for selection for different types of eating quality; by contrast, both loci and their surrounding regions show low diversity in GJ. The $R c^{25}$ locus has very low diversity in all variety groups, with variable diversity in the surrounding regions in $\mathrm{XI}, \mathrm{cA}$ and $\mathrm{cB}$.

We compared SNP variation among TE-related genes, NTE-related genes, 1,021 genes with validated functions curated in the OGRO/ QTARO database ${ }^{26,27}$ and a subset of 78 domestication and agronomically relevant genes (Supplementary Data 4). Genetic diversity was reduced significantly $\left(P\right.$ value $\left.<10^{-12}\right)$ near OGRO-curated genes and was often more extreme across the 78-gene subset in each subpopulation (Fig. 2b) when compared with all genomic regions containing genes, which suggests there may have been selection for these genes.

\section{Structural variations}

Structural variations (SVs) were called for 3,010 accessions but we focused on 453 accessions with sequencing depths $>20 \times$ and mapping depths $>15 \times$, because genome coverage stabilized when sequencing depths exceeded 20× (Extended Data Fig. 4a, b). We identified 93,683 SVs, including 582 SVs larger than $500 \mathrm{~kb}$, with an average of 12,178 SVs per genome. The average sizes of the detected deletions, inversions and duplications are $5.3 \pm 0.6 \mathrm{~kb}, 127.1 \pm 19.4 \mathrm{~kb}$ and $105.1 \pm 22.7 \mathrm{~kb}$, respectively (Fig. 3a, Extended Data Fig. 4c and Supplementary Data 3 Table 2).

SVs showed very strong XI-GJ differentiation. On average, each XI accession differed from Nipponbare RefSeq by 14,754 SVs $(8,990$ translocations, 5,411 deletions, 188 inversions and 165 duplications), or $3.5 \times$ as many as in GJ accessions (Fig. 3a). On average, each cA or cB accession differed from Nipponbare RefSeq by 12,997 SVs and 7,892 SVs, respectively. The total SV sequence that differentiated two GJ accessions was about $22 \mathrm{Mb}$, whereas it reached $71 \mathrm{Mb}$ between XI and GJ accessions (Fig. 3b). Notably, 1,940 SVs disrupted protein-coding genes within GJ, whereas $>6,518$ occurred between XI and GJ accessions (Fig. 3c). The SV phylogenetic tree (based on 453 accessions) is similar to the SNP tree, and clearly separates XI, GJ, cA and cB accessions (Fig. 3d). Moreover, the 41,957 major-group-unbalanced SVs that 


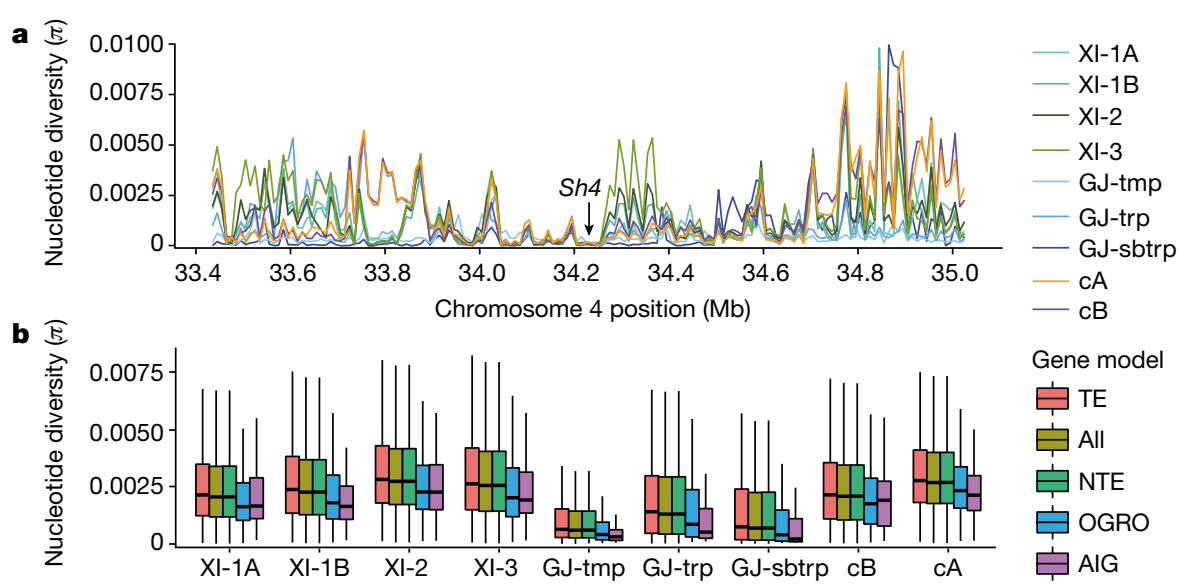

Fig. 2 Nucleotide diversity. a, Differential nucleotide diversity between subpopulations at the Sh4 locus on chromosome 4 using 10-kb sliding windows. $\mathbf{b}$, Box plots of the distribution of $\pi$ in 100-kb regions surrounding gene models across the genome. Box plots are shown for $k=9$ subpopulations for all $100-\mathrm{kb}$ windows (All) $(n=3,728$ in total) and those containing genes annotated as transposable elements (TE) $(n=3,305$

were distributed unevenly among XI, GJ, cA and cB accessions (Fig. 3e) accounted for $44.7 \%$ of all SVs and $41.0 \%$ of the 582 large SVs.

\section{Pan-genome and population differentiation}

The widespread SV and genome size variation (Supplementary Data 3 Tables 1 and 2) encouraged us to investigate the influence of PAVs on protein-coding genes across the 3K-RG. We first used a 'map-to-pan' strategy ${ }^{28}$ to build the species pan-genome (Extended Data Fig. 5a, b), by combining the Nipponbare RefSeq and non-redundant novel de novo assembled sequences; then, PAVs were determined by examining windows), NTE ( $n=3,709)$, from the OGRO/QTARO database (OGRO) $(n=828)$ and the subset of 78 domestication-related genes (AIG) $(n=61$ windows). Box plots show the median, box edges represent the first and third quartiles, and the whiskers extend to farthest data points within $1.5 \times$ interquartile range outside box edges.

gene-body and coding sequence (CDS) coverage of mapped reads for each accession.

We identified a total 268-Mb non-redundant novel sequences of length $>500$ bp with $<90 \%$ identity to Nipponbare RefSeq from assemblies of the 3,010 genomes, from which 12,465 novel full-length genes and several thousand novel genes with partial sequences were predicted. Nipponbare RefSeq genes and full-length novel genes could be merged into 23,876 gene families. The O. sativa core pan-genome was formed by $12,770(53.5 \%)$ gene families present in all 453 high-coverage genomes, $2,056(8.6 \%)$ without significant gene loss $>1 \%(P$ value $>0.05)$

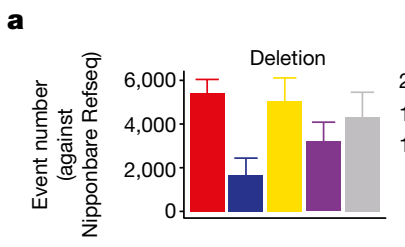

b
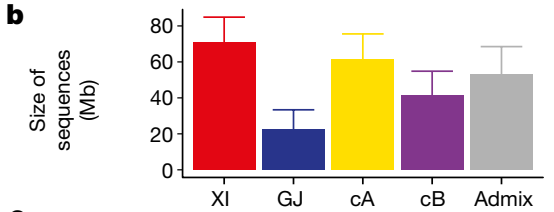

c

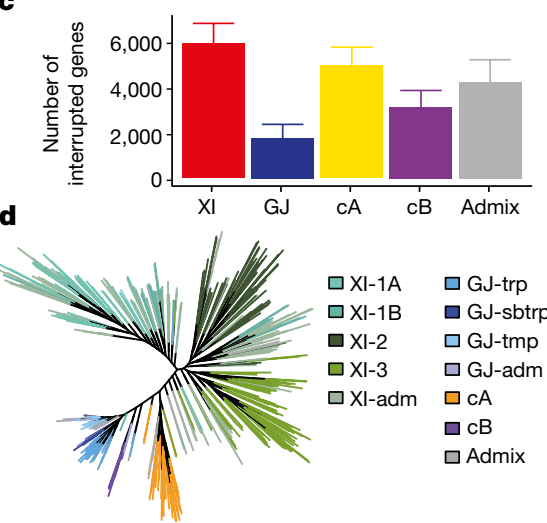

Fig. 3 Summary of SVs for the $\mathbf{4 5 3}$ high-coverage rice accessions. a, Number of deletions, duplications, inversions and translocations. b, Genome sizes affected by SVs. c, Numbers of genes affected (included or interrupted) by the SVs. d, Phylogenetic relationship of 453 rice accessions built from 10,000 randomly selected SVs. e, Characterization of the 42,207
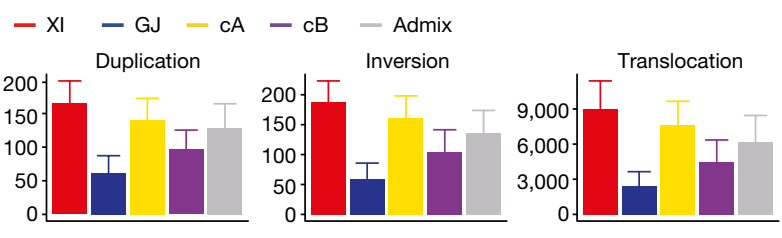

e

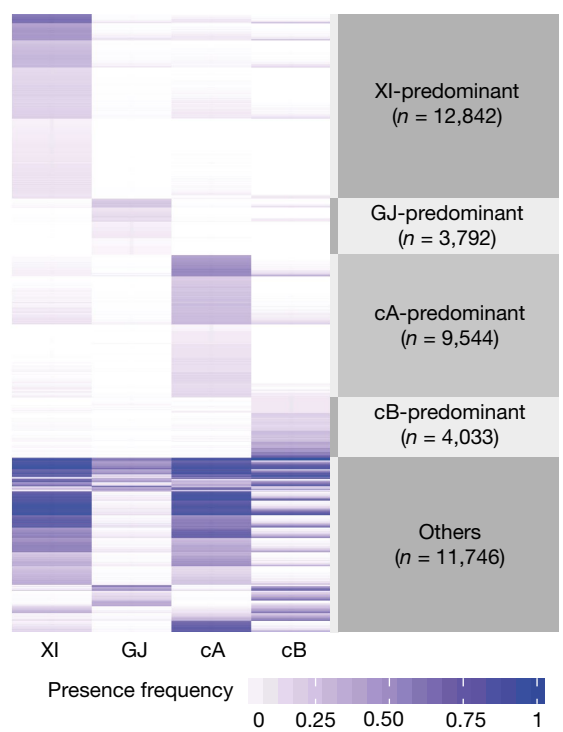

major-group-unbalanced SVs unevenly distributed among XI, GJ, cA and $\mathrm{cB}$ on the basis of two-sided Fisher's exact tests. Bar plots in $\mathbf{a}-\mathbf{c}$ are mean \pm s.d. and numbers of accessions in XI, GJ, cA, cB and admix are $303,92,33,10$ and 15 , respectively. 


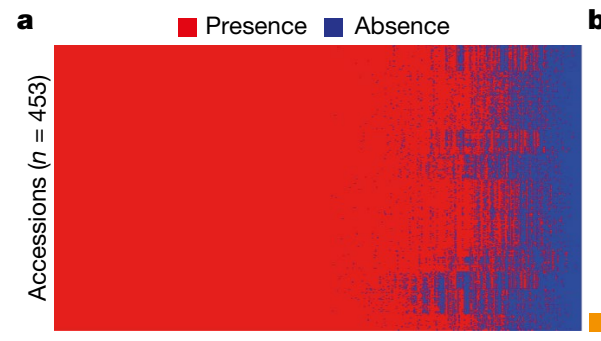

Gene families $(n=23,876)$

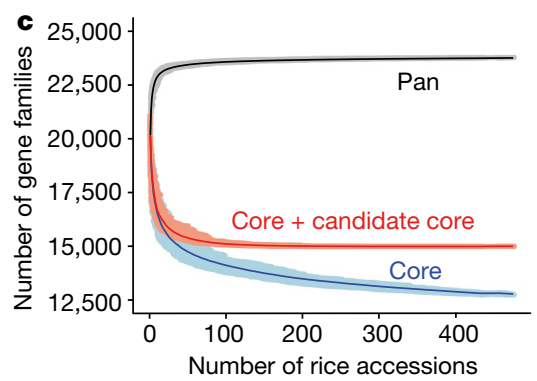

d

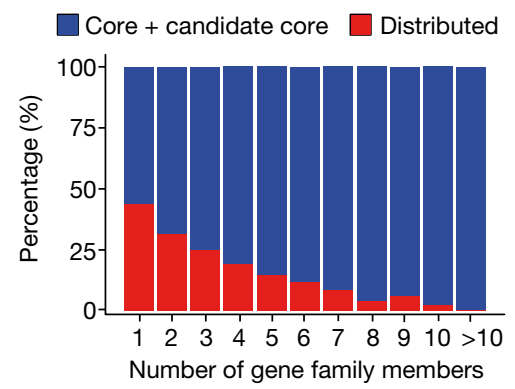

e

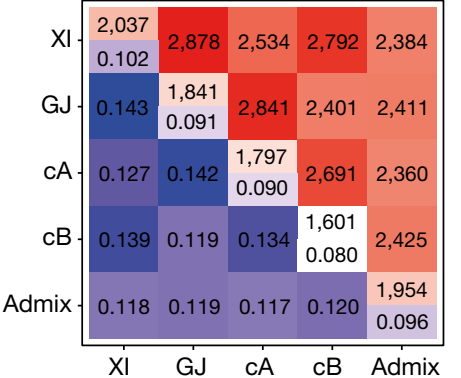

Fig. 4 | Pan-genome of O. sativa. a, Landscape of gene-family PAVs. Gene families were sorted by their occurrence and rice accessions were clustered with $k$-means method $(k=10)$. $\mathbf{b}$, Compositions of the pan-genome and an individual genome. $c$, Simulation of the pan-genome and core genome based on 500 randomizations of rice genome orders. d, Proportions of

in all major groups formed candidate core gene families, and the remaining 9,050 (37.9\%) comprised distributed gene families (Fig. 4a, b and Supplementary Data 3 Table 3). In silico simulation indicated these 9,050 gene families underestimate the distributed pan-genome (Fig. 4c). Hence, the O. sativa pan-genome consists of between 12,770 and approximately 14,826 (53.5\% to about $62.1 \%)$ core gene families, and at least 9,050 (37.9\%) distributed gene families: each accession contains between $63.4 \%$ and about $73.5 \%$ core gene families and at least $26.5 \%$ distributed gene families (Fig. $4 \mathrm{~b}$ ). The core gene families have more members (Fig. 4d) and represent essential gene families. Indeed, 5,476 (36.9\%) core or candidate core gene families are enriched in essential functions for growth, development and reproduction (using Gene Ontology, GO), whereas only 862 (9.5\%) of the distributed gene families could be annotated with GO terms, showing enrichment in regulation of immune and defence responses and ethylene metabolism (Extended Data Fig. 6a, b).

Pan-genome sequence coverage was evaluated using two new reference genomes ${ }^{29}$, IR 8 from the XI group and $\mathrm{N} 22$ from the cA group

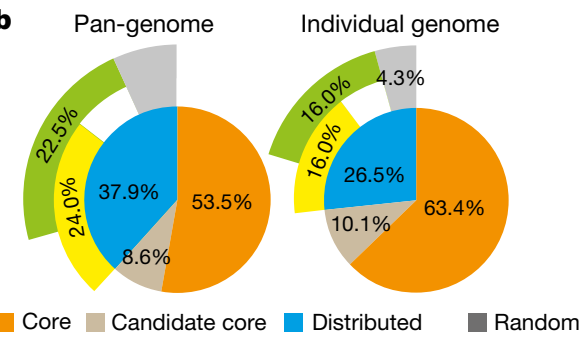

Major-group-unbalanced Subpopulation-unbalanced

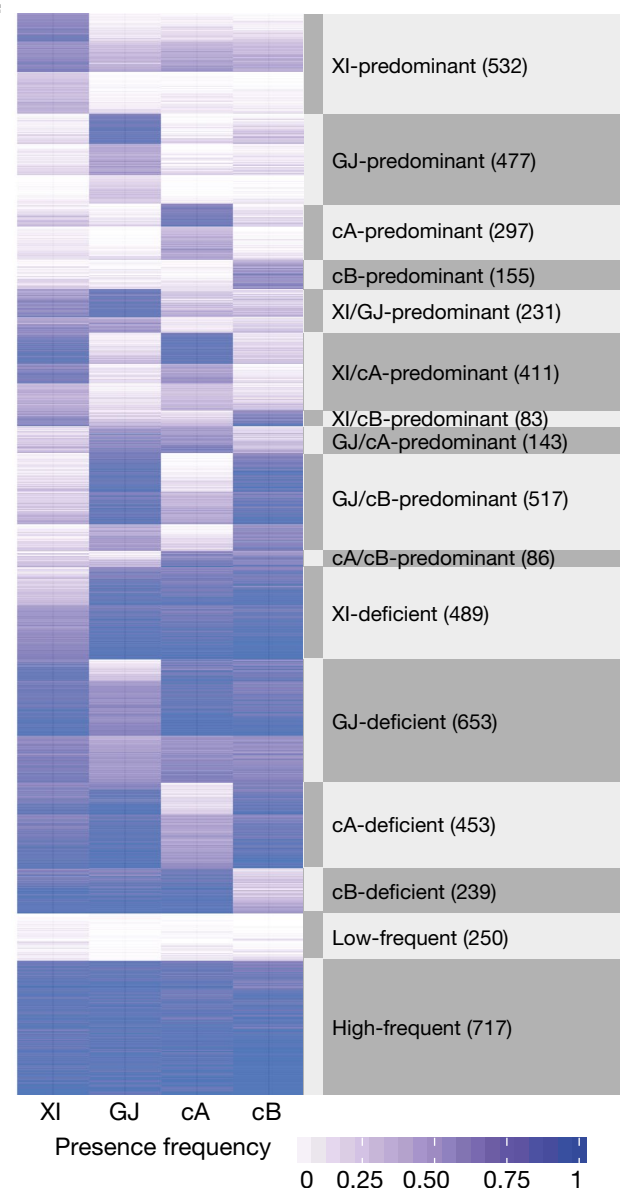

the core and distributed gene families binned by gene family sizes. e, The average number of gene families that are different between two accessions. f, Characterization of 5,733 major-group-unbalanced gene families detected by two-sided Fisher's exact tests.

(Supplementary Data 3 Table 4). We found $98.4 \%$ of the IR 8 and $98.6 \%$ of the $\mathrm{N} 22$ genome sequences could be mapped to the pangenome, whereas only $94.3 \%$ and $94.0 \%$ could be found in Nipponbare RefSeq. By comparing pan-genome data with high-quality XI reference genomes of Zhenshan 97 and Minghui $63^{30}$, approximately $25 \%$ of the novel genes were shorter owing to gene predictions from fragmented sequences (Extended Data Fig. 5c, d). Novel gene assemblies were validated by mapping raw reads of the 453 high-coverage genomes to the 12,465 novel genes; 11,792 genes $(94.6 \%)$ had $>95 \%$ CDS and $>85 \%$ gene-body coverages were present in at least two rice lines. By comparison, $99.9 \%$ of Nipponbare RefSeq annotated genes were detected in the 453 high-coverage genomes (Extended Data Fig. 5e). Approximately $30 \%$ of the full-length novel genes were expressed with $>1$ read per kilobase per million reads in one or more of the 226 publicly available RNA sequencing datasets ${ }^{31}$ (Extended Data Fig. 5f, g). Further, benchmarking universal single-copy orthologues $^{32}$ evaluation suggested little redundancy in predicted genes (Extended Data Fig. 5h). 
Analyses of the PAVs of genes (or gene families) were able to distinguish the major varietal groups, and show that there is considerable variation among and within subpopulations (Extended Data Fig. $7 \mathrm{a}-\mathrm{d})$. On average, major group accessions differ by about 4,000 (approximately 10\%) genes and about 2,000 (approximately 10\%) gene families, whereas XI and GJ accessions differ by more than 6,144 (about 14.9\%) genes and 2,878 (14.3\%) gene families (Fig. 4e and Extended Data Fig. 7e). The GJ pan-genome has 23,167 gene families comprising 46,115 genes, which makes it $1.9 \%$ smaller than XI in terms of gene families and $2.5 \%$ smaller in terms of genes. However, all GJ accessions have 240 core gene families (1,594 genes) in common, four times as many as in XI (Extended Data Fig. 7f). In addition, 5,733 major-group-unbalanced gene families were more frequent in some populations but lower in others, including hundreds of XI- and GJ-predominant gene families (Fig. 4f). Moreover, we identified 4,270 XI and 1,384 GJ subpopulation-unbalanced gene families, showing variation between subpopulations within each major group (Extended Data Fig. 7g).

\section{Evolution and domestication of rice}

To gain insights into the evolutionary history of the rice pan-genome, gene and gene family ages were estimated by aligning protein sequences to the NR protein database (ftp://ftp.ncbi.nlm.nih.gov/blast/db/) partitioned into 13 taxonomic levels (Extended Data Fig. 8a, b). We observed that: (1) new genes and gene families evolved at alternating rates from phylostratum 1 (PS1) (approximately 3.6 billion years ago) to the emergence of the terminal PS13 clade containing O. sativa (about 1.5 million years ago); (2) there was an explosive emergence of new genes accompanying the appearance of Oryza at PS12; (3) core genes tended to be more ancient, and most novel genes or gene families were younger and shorter (Extended Data Fig. 8c, d), consistent with recent reports for other species ${ }^{33}$; $(4)$ significantly $(P$ value $<0.001)$ higher SNP variation occurred in distributed genes than in core genes $(0.0325$ versus 0.0142 SNPs per base) (Extended Data Fig. 8e); and (5) a significantly $(P$ value $<0.001)$ higher proportion of core genes were under negative selection as compared with those in the Nipponbare RefSeq (Extended Data Fig. 8f).

Regarding $O$. sativa domestication, we constructed haplotype plots for nine important domestication genes- $R c^{25}, B h 4^{34}, P R O G 1^{35}$, $O s C 1^{36}, S h 4^{19}, W x^{23}, G S 3^{37}, q S H 1^{20}$ and $q S W 5^{38}$ (Fig. $5 \mathrm{a}-\mathrm{c}$ and Extended Data Fig. 9). Although a large number of XI samples carry an allele found in GJ, many XI accessions carry alleles at each of these loci that are absent in GJ (Fig. $5 \mathrm{~d}$ ). In fact, about $70 \%$ of XI accessions do not carry GJ introgressions in at least four genes, and only one XI sample (out of 1,789) had introgressed GJ haplotypes at all nine genes. This observation supports a model of independent domestication of some of the XI pool, rather than the simpler GJ-to-XI introgression hypothesis ${ }^{2}$. Furthermore, the 14-bp deletion in $R c^{25}$ for domesticated white pericarp was found in several XI lines that carried non-introgressed haplotypes (Extended Data Fig. 9), which suggests independent selection in part of the XI gene pool before introgression of the GJ haplotype became widespread in XI.

\section{Utility of the $3 \mathrm{~K}-\mathrm{RG}$ panel}

We demonstrated the use of the $3 \mathrm{~K}-\mathrm{RG}$ genomes and SNPs for trait mapping analyses for the highly heritable traits of grain length, grain width and bacterial blight resistance (Supplementary Notes). Major peaks for grain length with significantly $\left(P\right.$ value $\left.<10^{-10}\right)$ associated markers are on chromosomes 1, 3, 5, 6 and 7, and minor peaks are on chromosomes 4, 9, 10 and 11 (Extended Data Fig. 10a). Major peaks for grain width are found on chromosomes 1 and 5, with minor peaks on chromosomes 3 and 9 (Extended Data Fig. 10b). Genome-wide association study (GWAS) peaks were concordant with known loci, including $G S 3^{37}, G W 5^{39}$, and $q G L 7^{40}$ for grain length, and $G W 5$ for grain width. For grain width, the chromosome 9 novel peak coincides with $O s F D 1^{41}$, which codes for a bZIP transcription factor involved in flowering time and developmental plasticity (its pleiotropic regulatory
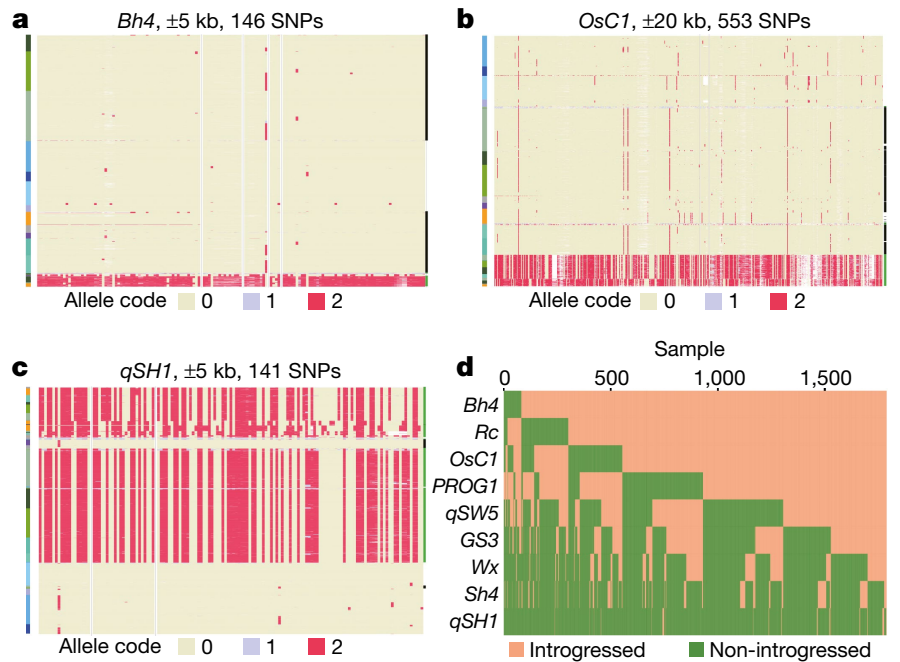

Fig. 5 | Haplotype analyses and introgression. a-c, Haplotypes around the domestication genes $B h 4(\mathbf{a}), \mathrm{OsCl}(\mathbf{b})$ and $q \mathrm{SH} 1(\mathbf{c})$. Rows correspond to samples and columns correspond to SNPs. Grey vertical lines mark the gene position. Left colour bar represents the $k=9$ subpopulations. Right colour bar shows introgression status of the XI samples (green, no introgression; black, putative introgression from GJ). d, A heat map showing results of an introgression test of 1,789 XI samples at each of the nine domestication genes. $y$ axis, genes; $x$ axis, XI samples.

function may therefore also affect grain width). Twelve peaks were detected for bacterial blight resistance to strain C5 of Xanthomonas oryzae, with the largest clustered around the resistance gene $\mathrm{Xa26}{ }^{42}$ on chromosome 11 (Extended Data Fig. 10c). Moreover, correlation between gene PAVs and plant height detected the well-known green revolution gene $(s d 1)$ as the first-ranked candidate. $s d 1$ is classified as a distributed gene-caused by an approximately 385-bp deletionand is significantly ( $P$ value $<10^{-20}$ ) associated with greatly reduced plant height; it was absent most frequently in XI-1A and XI-1B varieties (Extended Data Fig. 11).

\section{Discussion}

We characterized genetic variation in the 3,010 sequenced accessions of $O$. sativa and found a high level of genetic diversity in rice. Although the $3 \mathrm{~K}-\mathrm{RG}$ analysis is expected to identify nearly all polymorphisms with MAF $>1 \%$, our simulations suggest that it includes $<40 \%$ of rare bi-allelic SNPs (MAF $<1 \%$ ) in the International Rice Gene bank at the International Rice Research Institute (Extended Data Fig. 1c). We also characterized structural variation, and found that the average number of SVs between pairs of XI genomes $(>12,000)$ was similar to that between two high-quality reference XI genomes ${ }^{30}$. The vast majority were deletions and translocations distributed across the genome (Extended Data Fig. 4c). Mediumsized SVs $(\geq 500 \mathrm{~kb}$ ) were mostly inversions and duplications, and a large percentage of them (37.9\%) occur differentially between XI and GJ. We speculate that large numbers of SVs may contribute to the varying degrees of hybrid sterility and hybrid breakdown between $\mathrm{XI}$ and GJ accessions ${ }^{43}$. We also report pan-genome analyses for O. sativa, and the high numbers of PAVs highlight another component of within-species diversity for rice.

Our analysis brings more resolution to the within-species diversity of O. sativa (Extended Data Fig. 8e). Larger pan-genomes occur in XI than GJ accessions, but GJ accessions have more core genes than XI (Supplementary Data 3 Table 3), a result that was expected given the greater diversity within XI than GJ. This may relate to differences in eco-geographical distribution: GJ accessions experience harsher highaltitude and/or high-latitude environments, versus the less harsh but more diverse environments experienced by XI rice. Understanding the major group/subpopulation-core, -unbalanced and -predominant gene 
functions is expected to shed light on environmental adaptation of rice variety groups over thousands of years.

Although the $3 \mathrm{~K}-\mathrm{RG}$ population structure analyses based on SNPs and SVs were consistent with the five major groups that were previously known, additional subpopulations in the XI and GJ groups were identified and were suggestive of nine subpopulations that are correlated with geographic origin. Large numbers of SNPs, genes and gene families, and SVs were found to be unique to or predominant in single subpopulations. Varying patterns of diversity reduction across different rice subpopulations were observed in and around about 1,000 well-characterized genes. A closer look at patterns of haplotype sharing at domestication genes suggests that not all 'domestication' alleles came to XI from GJ. Taken together, our results-combined with archaeological evidence of XI cultivation for $>9,000$ years in both India and China ${ }^{44,45}$ - support multiple independent domestications of $O$. sativa.

Our 3K-RG analysis highlights the genetic diversity that exists in rice germplasm repositories, and the usefulness of establishing a digital gene bank in which all accessions can be sequenced and catalogued. For example, we estimate that sequencing the rest of the gene bank of the International Rice Research Institute may enable the identification of $>27$ million additional SNPs (Extended Data Fig. 1d). The next challenge will be to examine associations of the $3 \mathrm{~K}-\mathrm{RG}$ genetic variation with agriculturally relevant phenotypes measured under multiple field and laboratory environmental conditions; this will guide and accelerate rice breeding by identifying genetic variation that will be useful in breeding efforts and future sustainable agriculture.

\section{Online content}

Any Methods, including any statements of data availability and Nature Research reporting summaries, along with any additional references and Source Data files, are available in the online version of the paper at https://doi.org/10.1038/s41586018-0063-9.

Received: 27 November 2016; Accepted: 28 February 2018; Published online 25 April 2018.

1. Seck, P. A., Diagne, A., Mohanty, S. \& Wopereis, M. C. Crops that feed the world 7: rice. Food Secur. 4, 7-24 (2012).

2. Huang, $X$. et al. A map of rice genome variation reveals the origin of cultivated rice. Nature 490, 497-501 (2012).

3. Li, L. F., Li, Y. L., Jia, Y., Caicedo, A. L. \& Olsen, K. M. Signatures of adaptation in the weedy rice genome. Nat. Genet. 49, 811-814 (2017).

4. Wang, H., Vieira, F. G., Crawford, J. E., Chu, C. \& Nielsen, R. Asian wild rice is a hybrid swarm with extensive gene flow and feralization from domesticated rice. Genome Res. 27, 1029-1038 (2017)

5. Ting, Y. Origination of the rice cultivation in China. J. College of Agric. Sun Yat-Sen University 7, 11-24 (1949)

6. Glaszmann, J.-C. Isozymes and classification of Asian rice varieties. Theor. Appl. Genet. 74, 21-30 (1987).

7. Garris, A. J., Tai, T. H., Coburn, J., Kresovich, S. \& McCouch, S. Genetic structure and diversity in Oryza sativa L. Genetics 169, 1631-1638 (2005).

8. Chang, T.-T. The origin, evolution, cultivation, dissemination, and diversification of Asian and African rices. Euphytica 25, 425-441 (1976).

9. Han, B. \& Xue, Y. Genome-wide intraspecific DNA-sequence variations in rice. Curr. Opin. Plant Biol. 6, 134-138 (2003).

10. Agrama, H. A., Yan, W., Jia, M., Fjellstrom, R. \& McClung, A. M. Genetic structure associated with diversity and geographic distribution in the USDA rice world collection. Nat. Sci. 2, 247-291 (2010).

11. Allender, C. The Second Report on the State of the World's Plant Genetic Resources for Food and Agriculture. Rome: Food and Agriculture Organization of the United Nations (2010), 370 pp., ISBN 978-92-5-106534-1. Exp. Agric. 47, (574-574 (2011)

12. The 3,000 rice genomes project. The 3,000 rice genomes project. Gigascience 3, 7 (2014).

13. Golicz, A. A. et al. The pangenome of an agronomically important crop plant Brassica oleracea. Nat. Commun. 7, 13390 (2016).

14. Vernikos, G., Medini, D., Riley, D. R. \& Tettelin, H. Ten years of pan-genome analyses. Curr. Opin. Microbiol. 23, 148-154 (2015)

15. Kawahara, Y. et al. Improvement of the Oryza sativa Nipponbare reference genome using next generation sequence and optical map data. Rice (N. Y.) 6, 4 (2013).

16. Xu, X. et al. Resequencing 50 accessions of cultivated and wild rice yields markers for identifying agronomically important genes. Nat. Biotechnol. 30, 105-111 (2012).

17. Alexandrov, N. et al. SNP-Seek database of SNPs derived from 3000 rice genomes. Nucleic Acids Res. 43, D1023-D1027 (2015).
18. McCouch, S. R. et al. Open access resources for genome-wide association mapping in rice. Nat. Commun. 7, 10532 (2016).

19. Li, C., Zhou, A. \& Sang, T. Rice domestication by reducing shattering. Science 311, 1936-1939 (2006)

20. Konishi, S. et al. An SNP caused loss of seed shattering during rice domestication. Science 312, 1392-1396 (2006).

21. Sasaki, A. et al. Green revolution: a mutant gibberellin-synthesis gene in rice. Nature 416, 701-702 (2002).

22. Chandler, R. F. Jr. in Physiological Aspects of Crop Yield (ed. Dinauer, R.C.) (Crop Science Society of America, Madison, 1969).

23. Wang, Z. Y. et al. The amylose content in rice endosperm is related to the post-transcriptional regulation of the waxy gene. Plant J. 7, 613-622 (1995).

24. Chen, S. et al. Badh2, encoding betaine aldehyde dehydrogenase, inhibits the biosynthesis of 2-acetyl-1-pyrroline, a major component in rice fragrance. Plant Cell 20, 1850-1861 (2008)

25. Sweeney, M. T., Thomson, M. J., Pfeil, B. E. \& McCouch, S. Caught red-handed: Rc encodes a basic helix-loop-helix protein conditioning red pericarp in rice. Plant Cell 18, 283-294 (2006).

26. Yamamoto, E., Yonemaru, J., Yamamoto, T. \& Yano, M. OGRO: The overview of functionally characterized genes in rice online database. Rice (N. Y.) 5, 26 (2012).

27. Yonemaru, J.-I. et al. Q-TARO: QTL annotation rice online database. Rice (N. Y.) 3, 194-203 (2010).

28. $\mathrm{Hu}, \mathrm{Z}$. et al. EUPAN enables pan-genome studies of a large number of eukaryotic genomes. Bioinformatics 33, 2408-2409 (2017).

29. Stein, J. C. et al. Genomes of 13 domesticated and wild rice relatives highlight genetic conservation, turnover and innovation across the genus Oryza. Nat. Genet. 50, 285-296 (2018).

30. Zhang, J. et al. Extensive sequence divergence between the reference genomes of two elite indica rice varieties Zhenshan 97 and Minghui 63. Proc. Natl Acad. Sci. USA 113, E5163-E5171 (2016).

31. The IC4R Project Consortium. Information commons for rice (IC4R). Nucleic Acids Res. 44, D1172-D1180 (2015).

32. Simão, F. A., Waterhouse, R. M., Ioannidis, P., Kriventseva, E. V. \& Zdobnov, E. M. BUSCO: assessing genome assembly and annotation completeness with singlecopy orthologs. Bioinformatics 31, 3210-3212 (2015).

33. Wilson, B. A., Foy, S. G., Neme, R. \& Masel, J. Young genes are highly disordered as predicted by the preadaptation hypothesis of de novo gene birth. Nat. Ecol. Evol. 1, 0146 (2017)

34. Zhu, B. F. et al. Genetic control of a transition from black to straw-white seed hull in rice domestication. Plant Physiol. 155, 1301-1311 (2011).

35. Tan, L. et al. Control of a key transition from prostrate to erect growth in rice domestication. Nat. Genet. 40, 1360-1364 (2008).

36. Saitoh, K., Onishi, K., Mikami, I., Thidar, K. \& Sano, Y. Allelic diversification at the $\mathrm{C}(\mathrm{OsC1}$ ) locus of wild and cultivated rice: nucleotide changes associated with phenotypes. Genetics 168, 997-1007 (2004).

37. Fan, C. et al. GS3, a major QTL for grain length and weight and minor QTL for grain width and thickness in rice, encodes a putative transmembrane protein Theor. Appl. Genet. 112, 1164-1171 (2006).

38. Shomura, A. et al. Deletion in a gene associated with grain size increased yields during rice domestication. Nat. Genet. 40, 1023-1028 (2008).

39. Weng, J. et al. Isolation and initial characterization of GW5, a major QTL associated with rice grain width and weight. Cell Res. 18, 1199-1209 (2008).

40. Wang, Y. et al. Copy number variation at the GL7 locus contributes to grain size diversity in rice. Nat. Genet. 47, 944-948 (2015).

41. Taoka, K. et al. 14-3-3 proteins act as intracellular receptors for rice $\mathrm{Hd} 3 \mathrm{a}$ florigen. Nature 476, 332-335 (2011).

42. Sun, $X$. et al. Xa26, a gene conferring resistance to Xanthomonas oryzae pv. oryzae in rice, encodes an LRR receptor kinase-like protein. Plant J. 37, 517-527 (2004).

43. Shen, R. et al. Genomic structural variation-mediated allelic suppression causes hybrid male sterility in rice. Nat. Commun. 8, 1310 (2017).

44. Liu, L., Lee, G.-A., Jiang, L. \& Zhang, J. Evidence for the early beginning (c. 9000 cal. BP) of rice domestication in China: a response. Holocene 17, 1059-1068 (2007).

45. Fuller, D. Q., Allaby, R. G. \& Stevens, C. Domestication as innovation: the entanglement of techniques, technology and chance in the domestication of cereal crops. World Archaeol. 42, 13-28 (2010).

46. Alexander, D. H., Novembre, J. \& Lange, K. Fast model-based estimation of ancestry in unrelated individuals. Genome Res. 19, 1655-1664 (2009).

Acknowledgements This work was supported by the 863 Program (2014AA10A601) and National Key R\&D Program (2016YFD0100904) from the Chinese Ministry of Science \& Technology, Bill \& Melinda Gates Foundation Project (OPP1130530), the Agricultural Science and Technology Innovation Program Cooperation and Innovation Mission (CAAS-ZDXT201800), CAAS Innovative Team Award, International S\&T Cooperation Program of China (2012DFB32280), Shenzhen Peacock Plan, National Key Technology Support Program (2015BAD01B02), Fundamental Research Funds for Central NonProfit of CAAS (Y2017CG21) and National Natural Science Foundation of China (31501291, 61472246 and 61272250). IRRI was supported by the CGIAR Research Program CRP 3.3 (Global Rice Science Partnership). We thank the High-Performance Computing Centers at Shanghai Jiao Tong University, Agricultural Genomics Institute of CAAS and State Key Laboratory of Agricultural Genomics, BGI-Shenzhen (2011DQ782025). We thank M. Roa of the Philippine Genome Center Core Facilities for Bioinformatics, Department 
of Science and Technology-Advanced Science and Technology Institute of the Philippines, CyVerse, and XSEDE for computing and bioinformatics support; $Z$. Chong from University of Alabama for help in running novoBreak; the AXA Chair Research Fund and the Bud Antle Endowed Chair for the sequence analysis of IR 8 and N 22 genomes; and Amazon Public Data for free hosting of the $3 K-R G$ analyses results.

Reviewer information Nature thanks M. Bevan, $\mathrm{H}$. Tang and the other anonymous reviewer(s) for their contribution to the peer review of this work.

Author contributions R.M., D.Ch., S.T., T.Z., R.R.F., F.Z., L.M., M.S., K.C.P., D.Co., J.D., Jiab.X., D.K., S.L., Y.L., M.W., W.H., A.P., I.D., V.J.U., F.N.B., J.R.M., Q.G., Y.N. Z.Y., N.A. and K.L.M. performed the SNP and GWAS analyses. Z.H., W.W., C.S., B.F., X.C., M.C., Y.Zho., Y.Zha., R.L., B.J., Jiny.L., X.H., Z.D., J.S., Jing L., J.T., D.Z., J.A., Jing L., C.We. and Zh.L. performed the pan-genome analyses. Z.W., M.L., W.W., S.T., Jian.X., H.Z., Y.G., X.Zhao, F.S., H.Y., Zi.L., X.Zhan., C.Wa., X.W., Jinj.L., X.F., Y.Y., Zh.L. and J.R. performed de novo genome assembly and the structural variation analyses. D.Co. and R.A.W. conducted all repeat analysis. D.K., J.Z.

D.Co., M.E.B.N., J.T., S.L. and R.A.W. sequenced, assembled and annotated the IR 8 and N 22 reference genomes. W.W., R.M., Z.H., D.Ch., S.T., Z.W., M.L., T.Z., R.R.F., F.Z., L.M., D.Co., J.-C.G., Jiay.L., H.L., R.A.W., R.S.H., J.R., G.Z., C.We., N.A., K.L.M. and Zh.L. interpreted data and wrote the manuscript.

Competing interests The authors declare no competing interests.
Additional information

Extended data is available for this paper at https://doi.org/10.1038/s41586-

018-0063-9.

Supplementary information is available for this paper at https://doi. org/10.1038/s41586-018-0063-9.

Reprints and permissions information is available at http://www.nature.com/ reprints.

Correspondence and requests for materials should be addressed to $Z \mathrm{~h}$.L. K.L.M., C.We., J.R., N.A., G.Z. and R.A.W.

Publisher's note: Springer Nature remains neutral with regard to jurisdictional claims in published maps and institutional affiliations.

(c) (i)

Open Access This article is licensed under a Creative Commons Attribution 4.0 International License, which permits use, sharing, adaptation, distribution and reproduction in any medium or format, as long as you give appropriate credit to the original author(s) and the source, provide a link to the Creative Commons license, and indicate if changes were made. The images or other third party material in this article are included in the article's Creative Commons license, unless indicated otherwise in a credit line to the material. If material is not included in the article's Creative Commons license and your intended use is not permitted by statutory regulation or exceeds the permitted use, you will need to obtain permission directly from the copyright holder. To view a copy of this license, visit http:// creativecommons.org/licenses/by/4.0/. 


\section{METHODS}

No statistical methods were used to predetermine sample size. The experiments were not randomized and investigators were not blinded to allocation during experiments and outcome assessments.

Sequencing data of the 3,000 Rice Genome project. The selection and sequencing of rice accessions have previously been described ${ }^{12}$. The SNPs/indels and SVs in 3,010 accessions were identified by mapping against the Nipponbare RefSeq, and the pan-genome sequence was created by integrating the Nipponbare RefSeq and non-redundant novel sequences derived from 3,010 rice assemblies. SV comparison and gene PAV analyses focused on 453 rice accessions with sequencing depth $>20 \times$ and mapping depth $>15 \times$ (Extended Data Figs. $4 a, 5 b$ ).

Detection of SNPs and indels. Reads were aligned to the Nipponbare RefSeq using BWA-MEM (release 0.7.10) ${ }^{47}$. The mapped reads were then sorted and duplicates were removed by Picard tools (release 1.119) (http://broadinstitute. github.io/picard/). The reads around indels were realigned by GATK RealignerTargetCreator and IndelRealigner package (release 3.2-2) ${ }^{48}$. The variants were called for each accession by the GATK UnifiedGenotyper (release 3.2-2) ${ }^{48}$ with 'EMIT-ALL-SITES' option. A joint genotyping step for comprehensive SNP union and filtering step was performed on the 3,010 emit-all-sites VCF files. A variant position is reported if at least one sample supports it with QUAL no less than 30. A total of 29,399,875 SNPs (27,024,796 are bi-allelic) and 2,467,043 indels (small insertions and deletions $<40 \mathrm{bp}$ ) were identified from the analyses of the genomes of 3,010 accessions. Three subsets of the 3K-RG Nipponbare SNPs were defined using the following filtering criteria: (1) a base SNP set of $\sim 17$ million SNPs created from the $\sim 27$ million high-quality bi-allelic SNPs by removing SNPs in which heterozygosity exceeds Hardy-Weinberg expectation for a partially inbred species, with inbreeding coefficient estimated as $1-H_{\text {obs }} / H_{\text {exp }}$, in which $H_{\text {obs }}$ and $H_{\text {exp }}$ are the observed and expected heterozygosity, respectively (detailed in Supplementary Notes); (2) a filtered SNP set of $\sim 4.8$ million SNPs created from the $\sim 17$-million-SNP base SNP set by removing SNPs with $>20 \%$ missing calls and MAF $<1 \%$; and (3) a core SNP set of SNPs derived from the filtered SNP set using a two-step linkage disequilibrium pruning procedure with PLINK ${ }^{49,50}$, in which SNPs were removed by linkage disequilibrium pruning with a window size of $10 \mathrm{~kb}$, window step of one SNP and $r^{2}$ threshold of 0.8 , followed by another round of linkage disequilibrium pruning with a window size of 50 SNPs, window step of one SNP and $r^{2}$ threshold of 0.8 .

Determining the effects of SNPs. The effects of all bi-allelic SNPs (low, medium and high effects) on the genome were determined based on the pre-built release 7.0 annotation from the Rice Genome Annotation Project (http://rice.plantbiology. msu.edu/) using SnpEff ${ }^{51}$ release 4.11, with parameters -v -noLog -canon rice7. Using sequence ontology terms, a low-effect SNP was classified as 'synonymous_ variant', 'splice_region_variant', 'initiator_codon_variant', '5_prime_UTR_ premature_start_codon_gain_variant' or 'stop_retained_variant'. A moderateeffect SNP was identified as a 'missense_variant' and a high-effect SNP as a 'start_ lost', 'stop_gained,' 'stop_lost', 'splice_donor_variant' or 'splice_acceptor_variant'. For indel effects, only indels with lengths that were not multiples of three were counted and SNPs overlapped with protein-coding regions (CDSs of RGAP $7^{15}$ genes) were considered as the most disruptive effects on genes. Results of the SNP and indel effect analysis are given in Supplementary Data 2 Tables 3, 4. We computed the SNP numbers (proportions) of rare SNPs and homozygous singletons for a 'typical genome' of a subpopulation as the median SNP number (proportion) of the SNPs in a given category among those genomes for that subpopulation (Supplementary Data 2 Table 5).

Population structure and SNP diversity. Multi-dimensional scaling analysis was performed using the ' $\mathrm{cmdscale}$ ' function in R, using the IBS distance matrix of the $3 \mathrm{~K}-\mathrm{RG}$ genomes computed with PLINK ${ }^{49,50}$ on the filtered SNP set. The same distance matrix was used to construct a phylogenetic tree by the unweighted neighbour-joining method, implemented in the R package phangorn ${ }^{52}$. The population structure of the $3 \mathrm{~K}-\mathrm{RG}$ dataset was analysed using ADMIXTURE software ${ }^{46}$ on the core SNP set (version 0.4, http://snp-seek.irri.org/download. zul). First, ADMIXTURE was run on 30 random 100,000-SNP subsets of the core SNP set with $k$ (the number of groups) ranging from 5 to 18 , and $k=9$ was chosen because it was the minimal value of $k$ to separate all previously known groups (cA, cB, XI, GJ-trp, GJ-tmp and part of GJ-sbtrp). With $k=9$, ADMIXTURE was then run again on the whole core SNP set nine times with varying random seeds; the Q-matrices were aligned using CLUMPP software ${ }^{53}$ and clustered on the basis of similarity. Then, the matrices belonging to the largest cluster were averaged to produce the final matrix of admixture proportions. Finally, the group membership for each sample was defined by applying the threshold of $\geq 0.65$ to this matrix. Samples with admixture components $<0.65$ were classified as follows. If the sum of components for subpopulations within the major groups XI and GJ was $\geq 0.65$, the samples were classified as XI-adm or GJ-adm, respectively, and the remaining samples were deemed 'fully' admixed (admix). Branches of the phylogenetic tree were coloured according to the $k=9$ admixture classification (Fig. 1).
We computed linkage disequilibrium decay in each subpopulation as follows. The value of $r^{2}$ was computed for each pair of SNPs of frequency $\geq 10 \%$ in the respective subpopulations that are separated by at most $300 \mathrm{~kb}$ using PLINK. The distances were binned into 1-kb bins (separately for each chromosome) and the median value of $r^{2}$ in each bin was taken. The medians for each chromosome were then averaged to produce a final $r^{2}$ estimate for the bin. We computed nucleotide $\operatorname{diversity~}(\pi)$ for non-overlapping $10-\mathrm{kb}$ and $100-\mathrm{kb}$ windows along the Nipponbare RefSeq by adopting an approach similar to VariScan ${ }^{54}$ for genome-wide DNA polymorphism analyses and implemented as a custom R script.

Detection of genomic SVs and population differentiation. Genomic-SV detection for each of the 3,010 rice accessions was performed using a customized version of novoBreak ${ }^{55}$ (https://sourceforge.net/projects/novobreak/?source=navbar) against the Nipponbare RefSeq. SVs inferred by no less than 3 reads were further filtered with the following conditions: (1) more than four supporting split reads or (2) no fewer than three discordant read pairs. We detected deletions, inversions and duplications with sizes between $100 \mathrm{bp}$ and $1 \mathrm{Mb}$, and translocations. Here, translocations were SVs with 'inter-chromosomal breakpoints'. All SVs that passed the filter criteria in the $3 \mathrm{~K}-\mathrm{RG}$ accessions were pooled together. Two adjacent SVs were identified as the same SV if their start and end positions varied no more than $1 \mathrm{~kb}$, and the overlapping region was more than $50 \%$ of the total size. The presence-absence matrix of SVs in each accession was built based on this pooled SV dataset. To obtain reliable SV comparison analysis results, we focused only on the 453 high-depth accessions (Extended Data Fig. 4a). Majorgroup-unbalanced SVs were determined by two-sided Fisher's exact test followed by Benjamini-Hochberg adjustment (false discovery rate $(\mathrm{FDR})<0.05)$, similar to the detection of major-group-unbalanced genes.

De novo assembly. A variation of SOAPdenovo $2^{56}$ (version r240) with customized $k$-mers was used to assemble the rice genomes. A $k$-mer value was initially set for each accession according to a linear model ${ }^{\mathrm{C}} \mathrm{K}=2 *$ int $\left(0.38^{*}\right.$ (sequencing depth) +10$)+1$ ', which was trained from 50 randomly selected rice accessions. The best $k$-mer value was decided by checking the N50 of the SOAPdenovo results. The command line for SOAPdenovo was 'SOAPdenovo-63mer (or SOAPdenovo-127mer) all -s configure_file (average insertion length set as 460 in the configure file) $-\mathrm{K} k$-mer $-\mathrm{R}-\mathrm{F}^{\prime}$ with iteration over different $k$-mers until N50 of the assembly with that $k$-mer is higher than those with ' $k$-mer +2 ' and ' $k$-mer -2 '. On average, we needed to run SOAPdenovo $\sim 3.94$ times for each rice accession. The quality of the genome assembly was evaluated for these contigs using QUAST version $2.3^{57}$

Sequencing and de novo assembly of IR 8 and $\mathrm{N} 22$ reference genomes. High molecular weight DNA was extracted from young leaves adopting the proto$\mathrm{Col}^{58}$ with minor modifications. The PacBio library was prepared following the 20-kb protocol (see 'User-Bulletin-Guidelines-for-Preparing-20-kb-SMRTbellTemplates document.pdf', available from https://www.pacb.com/support/ documentation/?fwp_documentation_search="PN\%20100-286-700-04") and was sequenced on an RSII sequencer with movie collection time of $6 \mathrm{~h}$. The raw data of N 22 and IR 8 were assembled with FALCON ${ }^{59}$ and $\mathrm{Canu}^{60}$, respectively. Contigs were polished twice with PacBio raw reads using Quiver (https://github. com/PacificBiosciences/GenomicConsensus) and the IR 8 assembly was further polished with $66 \times$ WGS $2 \times 150$-bp Illumina data using Pilon ${ }^{61}$. Polished contigs were assigned to pseudomolecules using Genome Puzzle Master ${ }^{62}$. Assembly statistics can be found in Supplementary Data 3 Table 4. IR 8 and N 22 were applied to evaluate the completeness and redundancy of the pan-genome.

Pan-genome construction. SOAPdenovo assembly for each accession was assessed by QUAST ${ }^{57}$ with Nipponbare RefSeq as the reference. From QUAST output, unaligned contigs longer than $500 \mathrm{bp}$ were retrieved and merged. CD-HIT version 4.6.1 ${ }^{63}$ was used to remove redundant sequences at a cutoff of $90 \%$ identity with the command '-c 0.9 -T $16-\mathrm{M} 50000$ '. For remaining sequences, all-versus-all alignments with BLASTN were carried out to ensure that these sequences had no redundancy. Next, various contaminants including Archaea, bacteria, viruses, fungi and metazoans were removed. The non-redundant sequences were aligned to the NT database (downloaded from NCBI, 26 July 2014) with BLASTN with parameters '-evalue 1e-5 -best_hit_overhang 0.25 -perc_identity 0.5 -max_target_seqs 10 '. Contigs of which the best alignments (considering $E$-values and identities) were not from Viridiplantae were considered as contaminants and were filtered out. The remaining contigs formed the non-redundant novel sequences. The rice species pan-genome was then generated by combining the Nipponbare RefSeq and non-redundant novel sequences.

Annotation of the pan-genome. The gene-transcript annotation of the Nipponbare RefSeq was downloaded from the Rice Annotation Project ${ }^{64}$, and if a protein-coding gene contained multiple transcripts only the transcript with the longest open reading frame was selected as the representative for the gene. Protein-coding genes on novel sequences were predicted using MAKER ${ }^{65}$, a gene prediction tool combining ab initio predictions, expression evidence and protein homologies. In detail, repeats were first masked (soft mask for low-complexity 
repeats) with RepeatMasker (www.repeatmasker.org) and RepeatRunner ${ }^{66}$. Two ab initio predictors, $\mathrm{SNAP}^{67}$ and AUGUSTUS ${ }^{68}$, were called by MAKER ${ }^{65}$ to predict gene models with their default parameters for rice. All rice expressed sequence tags (ESTs) were downloaded from GenBank (15 December 2014) and were aligned to the novel sequences with BLASTN. All rice proteins were downloaded from NCBI (15 December 2014) and were aligned to the novel sequences with BLASTX. To obtain more informative alignments, Exonerate ${ }^{69}$ was used to realign each sequence identified by BLAST around splice sites. EVidenceModeller ${ }^{70}$ was used to combine and refine the ab initio predictions with RNA and protein evidence. Incomplete gene models were removed before the consequent analysis.

Adjustment of predicted genes. We aligned the predicted transcripts against Nipponbare RefSeq to remove potential redundancy. Redundant genes were removed when the genes were clustered into gene families. However, when attempting to identify the number of novel genes, the redundant ones were removed first. We clustered all genes at a global identity of $95 \%$, and removed novel genes that were not representative of the group.

Evaluation of pan-genome redundancy. We ran BUSCO (benchmarking universal single-copy orthologues) v.2.0 $0^{32}$ on CX140 (a Nipponbare accession) assembly, Nipponbare RefSeq, CX368 (an N 22 accession) assembly, N 22 high-quality reference genome and the pan-genome sequences. Augustus-3.2.3 ${ }^{68}$ and hmmer-3.1b $\mathrm{b}^{71}$ were used for gene prediction in BUSCO. BUSCO was run with genome mode with embryophyta_odb9 as a reference.

Functional analysis. All protein sequences of pan-genome were extracted and aligned to the GO sequence database (http://geneontology.org/ on 4 April 2015) with BLASTP. Only alignments with $E$-values $<1 \times 10^{-5}$ and identity $>0.3$ were used. GO terms for each gene were estimated to be the same as those of its best-hit protein. In total, 20,842 (43.3\%) genes could be annotated. For a gene family, its GO terms are the non-redundant set of the GO terms of the genes within this gene family. Overall, 6,338 (26.5\%) gene families could be annotated. Enrichment of GO terms was carried out using the GOstats ${ }^{72}$ package in $\mathrm{R}$ with all gene families as the background.

Validation of the non-Nipponbare RefSeq genes. We verified the novel genes by multiple approaches. First, for each gene, we examined the number of accessions that possessed it. We mapped the sequencing reads to the pan-genome sequences. Genes with CDS coverage over 0.95 and gene-body coverage over 0.85 were considered to be present. Second, we verified the novel genes with 226 RNA sequencing experiments from 17 projects $^{30}$. RNA sequencing reads were first trimmed with Trimmomatic version $0.32^{73}$ with parameters 'ILLUMINACLIP:2:30:10 LEADING:20 TRAILING:20 SLIDINGWINDOW:4:20 MINLEN:36' and then aligned to the pan-genome sequences with a split-aligner HISAT2 version 2.0.1beta $^{74}$ using default parameters. The coverage of each gene was calculated with 'BEDtools coverage' in BEDtools suite version 2.17.075.

Gene family annotation. The genes were clustered to gene families with OrthoMCL version 2.0.9 ${ }^{76}$. All genes were extracted and translated into protein sequences and the protein sequences were compared by using all-by-all BLASTP $\left(E\right.$-value $\left.=1 \times 10^{-5}\right)$. OrthoMCL was applied to process the BLASTP output and cluster genes to gene families. Similarity of protein families was set to be 0.5 as suggested by a previous publication ${ }^{76}$.

Determination of gene presence or absence. We proposed a 'map-to-pan' strategy to determine gene presence or absence ${ }^{28}$. For the 453 accessions with high sequencing depth, although only about $60 \%-70 \%$ of their genomes can be de novo assembled (contig $\geq 500 \mathrm{bp}$ ), more than $98 \%$ of their genomes can be covered by short read mapping. This enabled the use of coverage of genes to determine their presence or absence. In practice, genes with CDS coverage over 0.95 and gene-body coverage over 0.85 were considered present. If one member of a gene family is present in a given rice accession, the gene family is considered as present. Determination of core and distributed genes or gene families. A core gene (or gene family) is a gene (or gene family) present in all rice accessions, and we further defined candidate core genes (or gene families) as those with loss rates not significantly larger than 0.01 in all major groups. We first examined whether a gene (or a gene family) is distributed (loss rate $>0.01$ ) in each type of O. sativa (XI, GJ, $\mathrm{cA}$ and $\mathrm{cB}$ ). Binomial tests (with a null hypothesis of loss rate $<0.01$ ) were carried out for each gene in each type. A $P$ value below 0.05 meant that this gene (or a gene family) was lost in a significant proportion of rice accessions and is a distributed gene (or gene family) of these subpopulations. If a gene (or a gene family) was not determined to be distributed in all types (and it was not core), it was considered to be a candidate core gene (or gene family) of O. sativa. Other genes (or gene families) were considered to be distributed.

Determination of major-group-unbalanced, subpopulation-unbalanced and random genes or gene families. Distributed genes (or gene families) were divided further into major-group-unbalanced, subpopulation-unbalanced and random genes (or gene families). Major-group-unbalanced genes (or gene families) are defined as genes (or gene families) that are unequally distributed among XI, GJ, $\mathrm{cA}$ and $\mathrm{cB}$ groups. A two-sided Fisher's exact test was used to determine whether the distribution of each gene (or gene family) is uniform. The $P$ values of all genes were calculated with the 'Fisher.test' function in $\mathrm{R}$ and were then adjusted with the Benjamini-Hochberg FDR method. Genes (or gene families) with FDR $<0.05$ were considered as major-group-unbalanced.

Subpopulation-unbalanced genes (or gene families) are defined as genes (or gene families) that are unequally distributed among subpopulations; thus, they can be divided into XI-subpopulation-unbalanced genes (or gene families) and GJ-subpopulation-unbalanced genes (or gene families). XI-subpopulationunbalanced genes (or gene families) are defined as genes (or gene families) that are unequally distributed among XI-1A, XI-1B, XI-2 and XI-3 subpopulations. GJ-subpopulation-unbalanced genes (or gene families) can be defined similarly. The same statistical methods for the major groups were applied to determine the distribution balance for subpopulations. We defined genes (or gene families) that are neither major-group-unbalanced nor subpopulation-unbalanced to be 'random' genes.

Gene and gene-family age. Gene ages were inferred with previously described methods ${ }^{77}$. The NR protein database was downloaded from NCBI (28 March 2015) and all protein sequences were grouped according to 13 taxonomic levels (PS1: Cellular organisms; PS2: Eukaryota; PS3: Viridiplantae; PS4: Streptophyta, Streptophytina; PS5: Embryophyta; PS6: Tracheophyta, Euphyllophyta; PS7: Spermatophyta; PS8: Magnoliophyta, Mesangiospermae; PS9: Liliopsida, Petrosaviidae, Commelinids, Poales; PS10: Poaceae; PS11: BOP clade; PS12: Oryzoideae, Oryzeae, Oryza; and PS13: O. sativa) based on NCBI taxonomy. Thirteen BLASTP databases were built for protein sequences from PS1 to PS13. All genes on pan-genome sequences were first translated into proteins, and were aligned to the 13 databases using BLASTP with $E$-values $<1 \times 10^{-5}$ and identity $>0.3$. The age of a gene was considered as the taxonomic level of the oldest aligned protein. Genes that failed to align to all databases were assigned gene ages of PS13 (O. sativa). Some PS13 genes were reassigned as PS12 genes if they could be covered by 446 wild rice genomes ${ }^{2}$ with both gene-body coverage $>0.95$ and CDS coverage $>0.95$. The age of a gene family was considered as the age of the oldest gene within the gene family.

Introgression test. To test whether an XI sample had a non-introgression haplotype at a locus, we defined a $D$-value for a sample $x$ as $D(x)=d(x, \mathrm{XI})-d(x, \mathrm{GJ})$, in which $d(x, \mathrm{XI})$ is the mean distance from sample $x$ to a XI sample at the given locus.

With no gene flow from GJ to XI and vice versa, the $D$-value is negative for XI and positive for GJ. On the other hand, if an XI sample shares a haplotype with a GJ sample, the $D$-value will be positive and close to the $D$-values of GJ samples. For an XI sample, we rejected the hypothesis of GJ introgression if its $D$-value was negative and less than the lower bound of the $99 \%$ confidence interval for the $D$-value of GJ samples, which was computed on the subset of GJ consisting of samples with a positive $D$-value, to exclude the effect of potential XI-to-GJ introgression.

Reporting summary. Further information on experimental design is available in the Nature Research Reporting Summary linked to this paper.

Code availability. Code for studying pan-genome and gene and gene family PAVs are now integrated and published as the EUPAN toolkit ${ }^{28}$. Tailored novoBreak-germline is available at https://sourceforge.net/projects/novobreak/? source $=$ navbar. Code for nucleotide diversity and SNP merging is available at https:/github.com/dchebotarov/3k-SNP-paper. All other code is available from the corresponding authors upon request.

Data availability. The BAM alignment file and variant calls in VCF format for each accession of the 3K-RG against Nipponbare RefSeq are freely downloadable from Amazon Public Data at https://aws.amazon.com/public-data-sets/3000-ricegenome/ and the Department of Science and Technology Advanced Science and Technology Institute of the Philippines (DOST-ASTI) IRODS site, as described on the 3K-RG project site (http://iric.irri.org/resources/3000-genomes-project). The SV and PAV data of $3 \mathrm{~K}-\mathrm{RG}$ are available in the figshare database ${ }^{78}$ (https:// doi.org/10.6084/m9.figshare.c.3876022.v1).

The following web tools are available for the mining, analysis and visualization of the 3K-RG dataset: SNP-Seek, http://snp-seek.irri.org; RMBreeding databases, http://www.rmbreeding.cn/index.php; rice cloud of genetic data public projects, http://www.ricecloud.org/; IRRI Galaxy, http://galaxy.irri.org/; and the 3,000 rice pan-genome browser ${ }^{79}$, http://cgm.sjtu.edu.cn/3kricedb/.

The $3 \mathrm{~K}-\mathrm{RG}$ sequencing data used for our analyses can be obtained via project accession PRJEB6180 from NCBI (https://www.ncbi.nlm.nih.gov/sra/?term= PRJEB6180), accession ERP005654 from DDBJ (https://www.ddbj.nig.ac.jp/index -e.html) and from the GigaScience Database (https://doi.org/10.5524/200001).

47. Li, H. \& Durbin, R. Fast and accurate short read alignment with BurrowsWheeler transform. Bioinformatics 25, 1754-1760 (2009).

48. McKenna, A. et al. The Genome Analysis Toolkit: a MapReduce framework for analyzing next-generation DNA sequencing data. Genome Res. 20, 1297-1303 (2010).

49. Purcell, S. et al. PLINK: a tool set for whole-genome association and populationbased linkage analyses. Am. J. Hum. Genet. 81, 559-575 (2007). 
50. Chang, C. C. et al. Second-generation PLINK: rising to the challenge of larger and richer datasets. Gigascience 4, 7 (2015).

51. Cingolani, P. et al. A program for annotating and predicting the effects of single nucleotide polymorphisms, SnpEff: SNPs in the genome of Drosophila melanogaster strain w1118; iso-2; iso-3. Fly (Austin) 6, 80-92 (2012)

52. Schliep, K. P. phangorn: phylogenetic analysis in R. Bioinformatics $27,592-593$ (2011).

53. Jakobsson, M. \& Rosenberg, N. A. CLUMPP: a cluster matching and permutation program for dealing with label switching and multimodality in analysis of population structure. Bioinformatics 23, 1801-1806 (2007).

54. Hutter, S., Vilella, A. J. \& Rozas, J. Genome-wide DNA polymorphism analyses using VariScan. BMC Bioinformatics 7, 409 (2006).

55. Chong, Z. et al. novoBreak: local assembly for breakpoint detection in cancer genomes. Nat. Methods 14, 65-67 (2017).

56. Luo, R. et al. SOAPdenovo2: an empirically improved memory-efficient short-read de novo assembler. Gigascience 1, 18 (2012).

57. Gurevich, A., Saveliev, V., Vyahhi, N. \& Tesler, G. QUAST: quality assessment tool for genome assemblies. Bioinformatics 29, 1072-1075 (2013).

58. Doyle, J. J. A rapid DNA isolation procedure for small quantities of fresh leaf tissue. Phytochem. Bull. 19, 11-15 (1987).

59. Chin, C.-S. et al. Phased diploid genome assembly with single-molecule real-time sequencing. Nat. Methods 13, 1050-1054 (2016).

60 . Koren, S. et al. Canu: scalable and accurate long-read assembly via adaptive k-mer weighting and repeat separation. Genome Res. 27, 722-736 (2017).

61. Walker, B. J. et al. Pilon: an integrated tool for comprehensive microbial variant detection and genome assembly improvement. PLOS ONE 9, e112963 (2014).

62. Zhang, J. et al. Genome puzzle master (GPM): an integrated pipeline for building and editing pseudomolecules from fragmented sequences. Bioinformatics 32, 3058-3064 (2016).

63. Fu, L., Niu, B., Zhu, Z., Wu, S. \& Li, W. CD-HIT: accelerated for clustering the next-generation sequencing data. Bioinformatics 28, 3150-3152 (2012).

64. Ohyanagi, H. et al. The Rice Annotation Project Database (RAP-DB): hub for Oryza sativa ssp. japonica genome information. Nucleic Acids Res. 34, D741-D744 (2006).
65. Holt, C. \& Yandell, M. MAKER2: an annotation pipeline and genome-database management tool for second-generation genome projects. BMC Bioinformatics 12, 491 (2011).

66. Smith, C. D. et al. Improved repeat identification and masking in Dipterans. Gene 389, 1-9 (2007).

67. Korf, I. Gene finding in novel genomes. BMC Bioinformatics 5, 59 (2004).

68. Stanke, M., Steinkamp, R., Waack, S. \& Morgenstern, B. AUGUSTUS: a web server for gene finding in eukaryotes. Nucleic Acids Res. 32, W309-W312 (2004).

69. Slater, G. S. C. \& Birney, E. Automated generation of heuristics for biological sequence comparison. BMC Bioinformatics 6, 31 (2005)

70. Haas, B. J. et al. Automated eukaryotic gene structure annotation using EVidenceModeler and the program to assemble spliced alignments. Genome Biol. 9, R7 (2008).

71. Finn, R. D. et al. HMMER web server: 2015 update. Nucleic Acids Res. 43, W30-W38 (2015).

72. Falcon, S. \& Gentleman, R. Using GOstats to test gene lists for GO term association. Bioinformatics 23, 257-258 (2007)

73. Bolger, A. M., Lohse, M. \& Usadel, B. Trimmomatic: a flexible trimmer for Illumina sequence data. Bioinformatics 30, 2114-2120 (2014).

74. Kim, D., Langmead, B. \& Salzberg, S. L. HISAT: a fast spliced aligner with low memory requirements. Nat. Methods 12, 357-360 (2015).

75. Quinlan, A. R. \& Hall, I. M. BEDTools: a flexible suite of utilities for comparing genomic features. Bioinformatics 26, 841-842 (2010).

76. Li, L., Stoeckert, C. J., Jr \& Roos, D. S. OrthoMCL: identification of ortholog groups for eukaryotic genomes. Genome Res. 13, 2178-2189 (2003).

77. Zhang, Y. E., Vibranovski, M. D., Landback, P., Marais, G. A. \& Long, M. Chromosomal redistribution of male-biased genes in mammalian evolution with two bursts of gene gain on the X chromosome. PLoS Biol. 8, e1000494 (2010).

78. $\mathrm{Hu}, \mathrm{Z}$. et al. Novel sequences, structural variations and gene presence variations of Asian cultivated rice. Sci. Data https://doi.org/10.1038/sdata. 2018.79 (2018).

79. Sun, C. et al. RPAN: rice pan-genome browser for $\sim 3,000$ rice genomes. Nucleic Acids Res. 45, 597-605 (2017). 
a

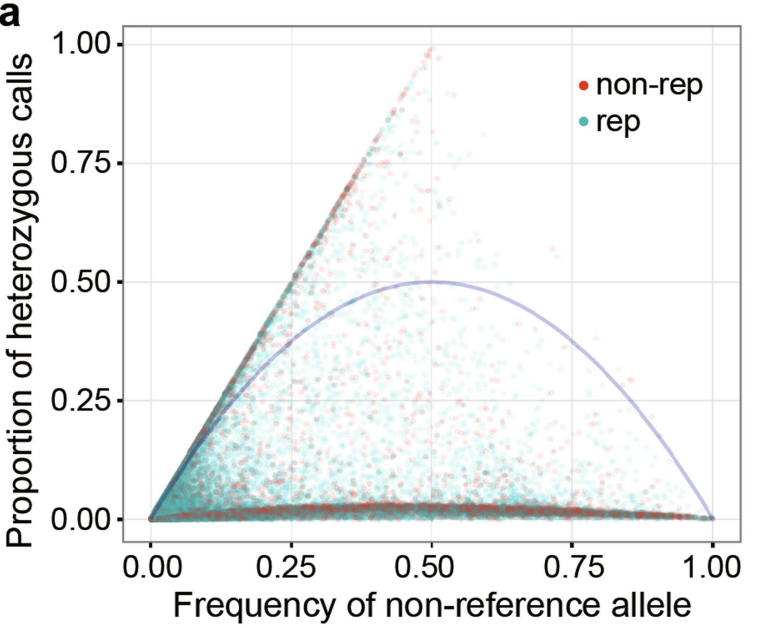

C

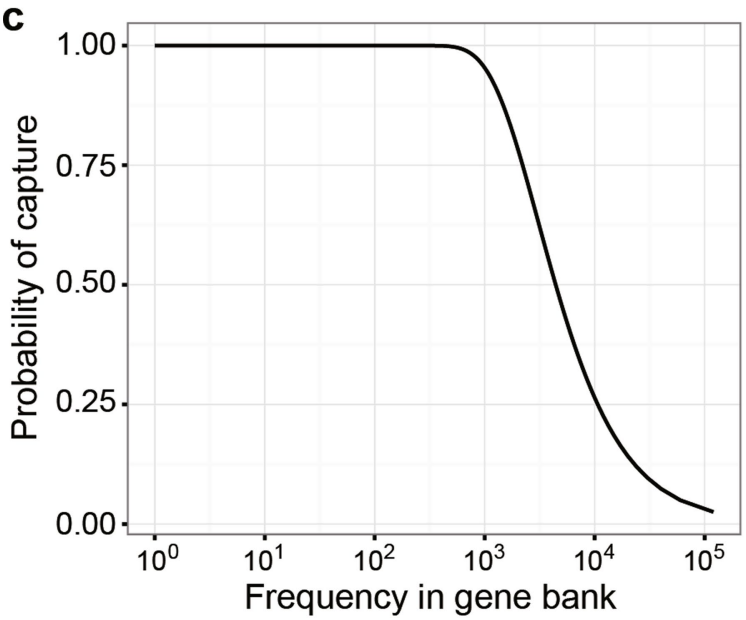

Extended Data Fig. 1 | SNP filtering, discovery rate, and projected discovery upon further sequencing. a, Proportion of heterozygous calls versus allele frequency. Each dot represents a SNP from a random sample of 100,000 SNPs. Blue curve shows theoretical Hardy-Weinberg equilibrium. The points have opacity of $5 \%$, such that regions with higher point densities are highlighted. The bulk of SNPs lie on the Hardy-

Weinberg equilibrium curve scaled by a factor of about 0.05 , which implies a Wright's inbreeding coefficient of $F=0.95$. b, The same plot with colour b
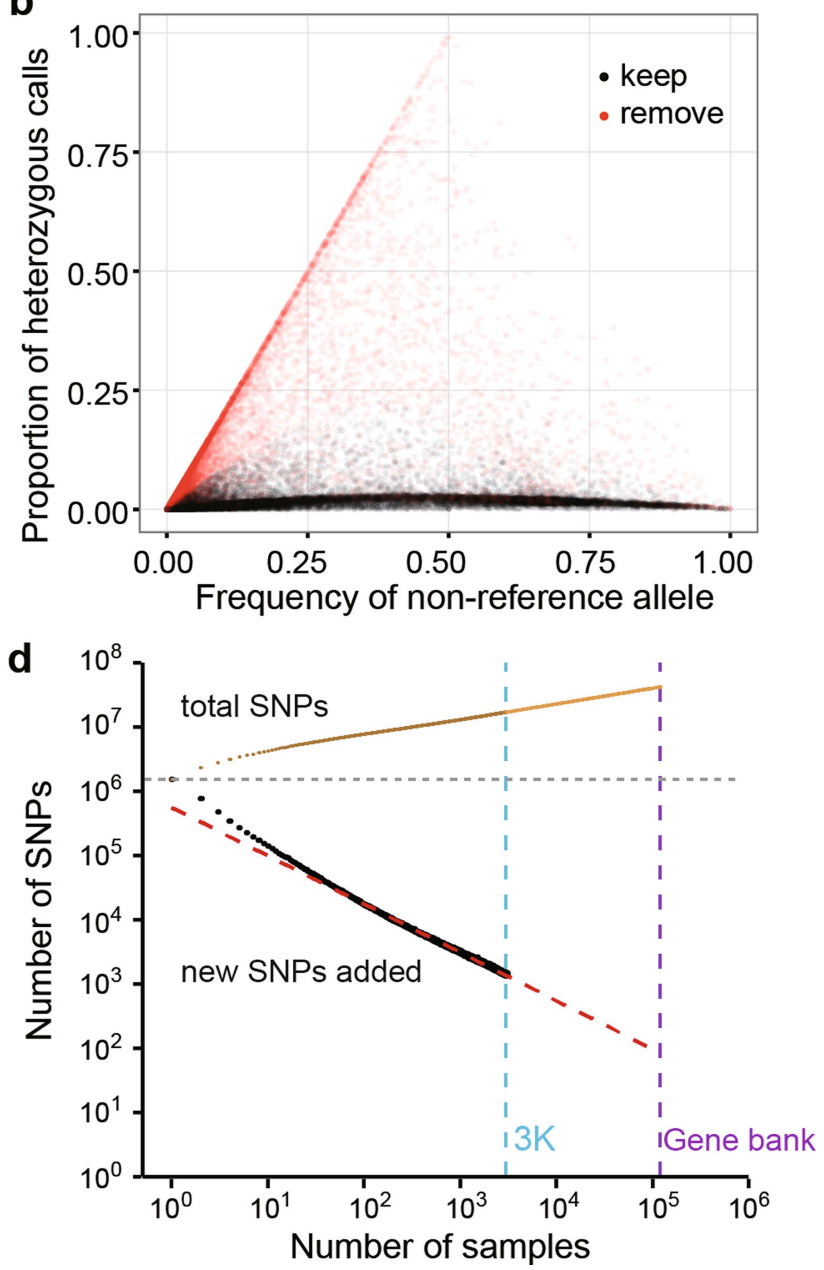

representing the outcome of filtering. The SNPs that are marked 'keep' (black) form the base SNP set.c, The estimated proportion of gene bank SNPs captured by $3 \mathrm{~K}-\mathrm{RG}$ samples, per frequency. The 3,010 samples capture more than $99.99 \%$ of gene-bank SNPs of frequency greater than $0.25 \%$. d, Projected new SNP discovery rate based on simulations. For a given number of samples ( $x$ axis), the graph shows estimated mean number of new SNPs discovered in the last sample. 

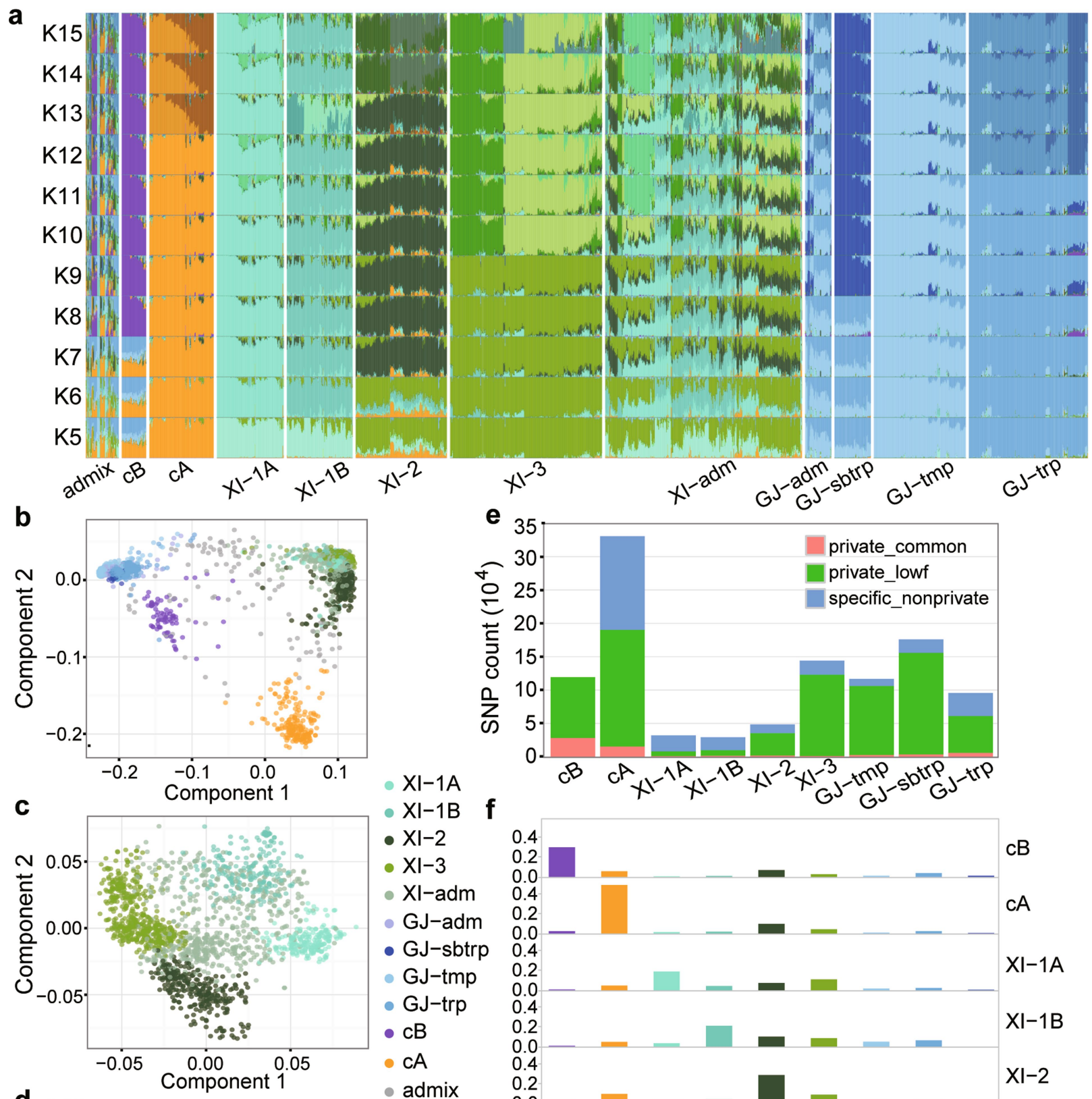

- XI-1A

- $\mathrm{XI}-1 \mathrm{~B} \quad \mathrm{f}$

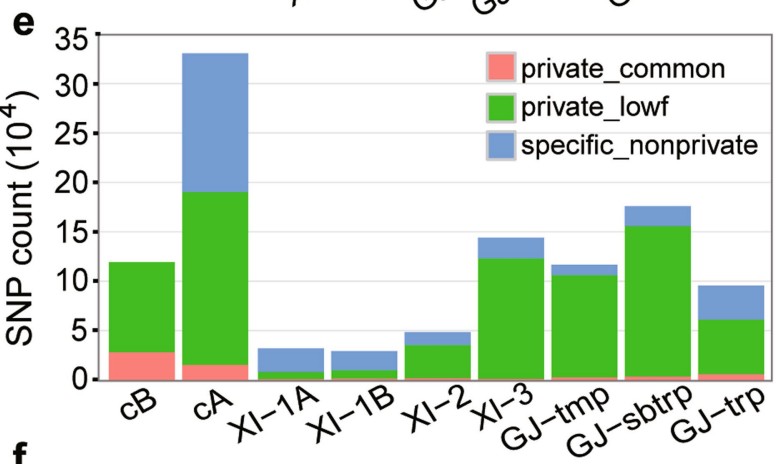

- $\mathrm{XI}-2$

- XI-3

- XI-adm

- GJ-adm

- GJ-sbtrp

- GJ-tmp

- GJ-trp

- $\mathrm{CB}$

- $c \mathrm{~A}$

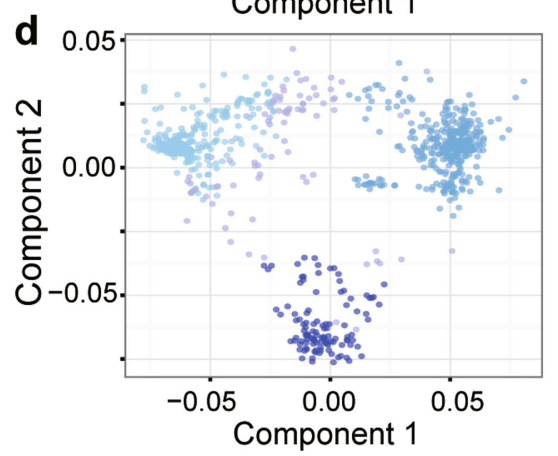

- admix

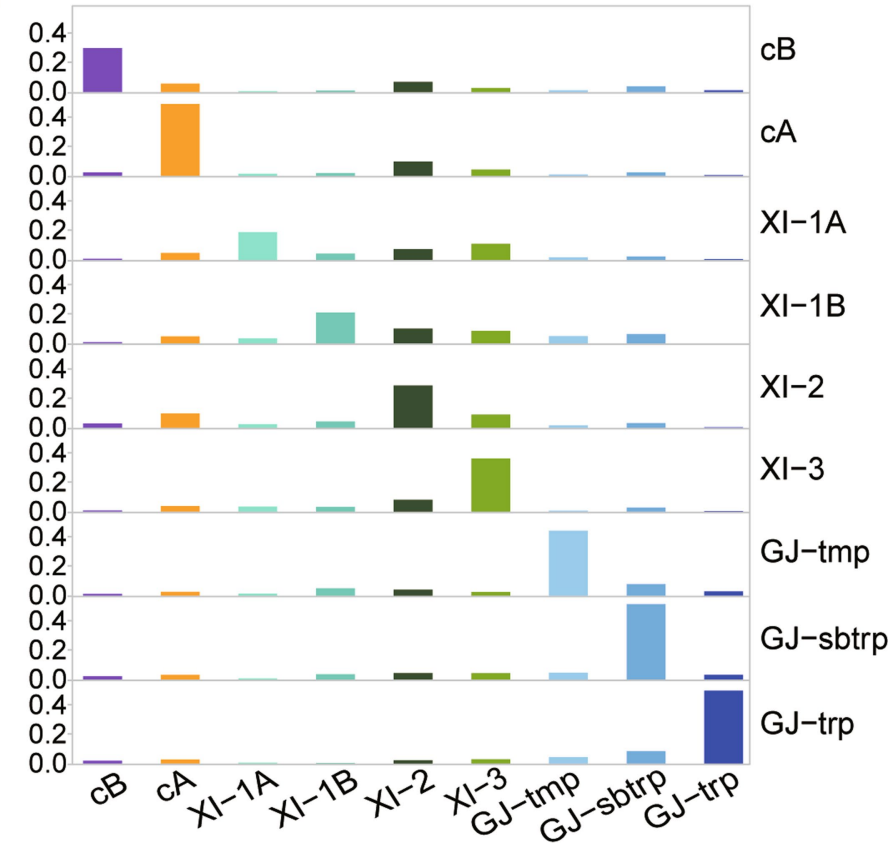

Extended Data Fig. 2 Population structure and subpopulation differentiation. a, ADMIXTURE analyses for $k=5$ to $k=15$.

b-d, Multidimensional scaling plots for all $(n=3,010)(\mathbf{b}), \mathrm{XI}(n=1,786)$

(c) and GJ $(n=849)(\mathbf{d})$ accessions. e, Private and specific SNPs in each

4 accessions in a subpopulation and not found in other subpopulations; population-specific alleles are common in the subpopulation $(\geq 20 \%)$ but of low frequency $(<2 \%)$ in others. f, Doubleton sharing - that is, SNPs shared by two accessions-within and between subpopulations, with values normalized by the sample sizes. 

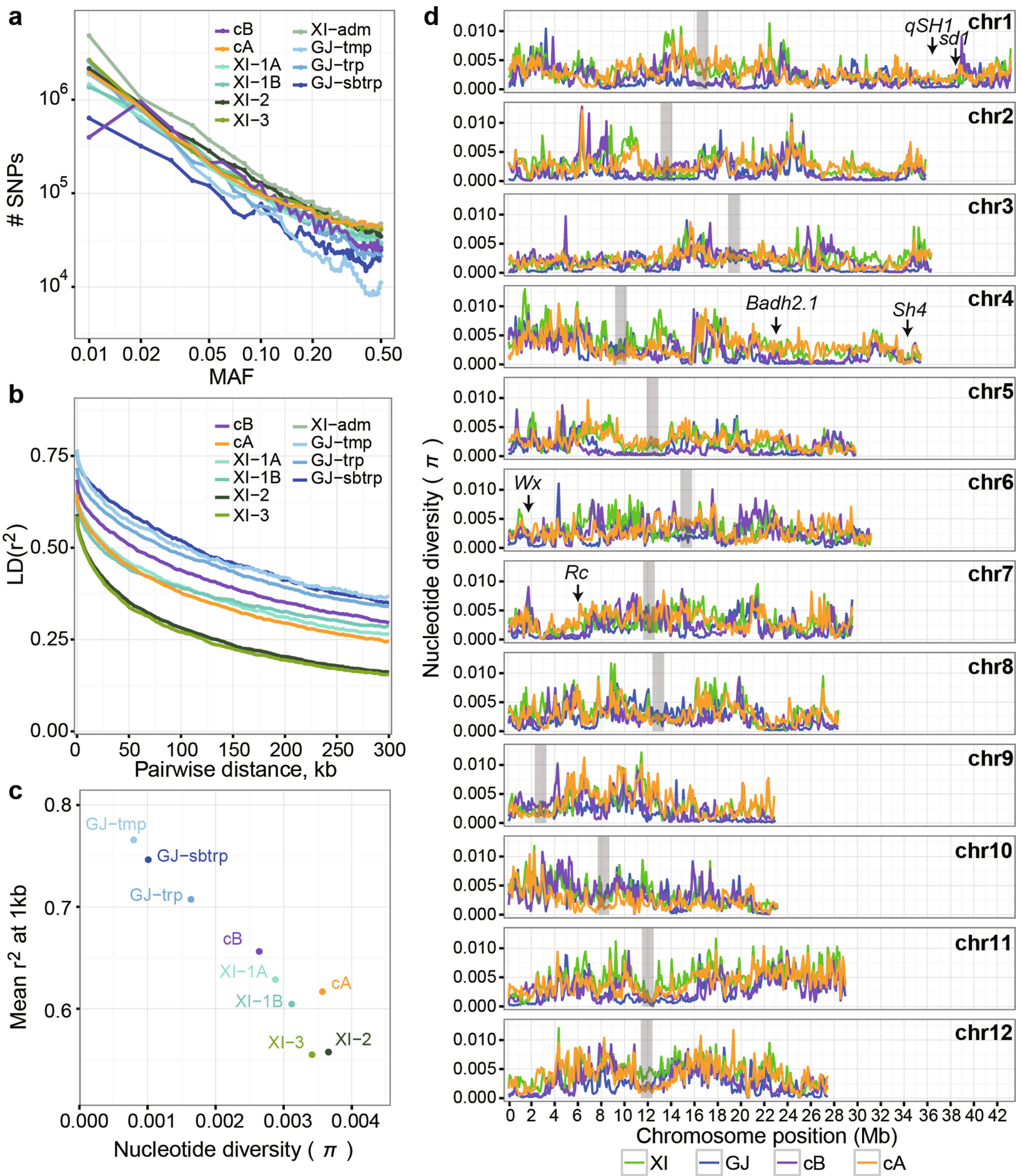

Extended Data Fig. 3 | Genetic diversity within subpopulations. a, MAF histogram. b, Genome-wide linkage disequilibrium. c, Nucleotide diversity for major groups (XI, GJ, cA and $\mathrm{cB}$ ) using 100-kb windows in which centromeric regions are highlighted in grey. versus linkage disequilibrium. d, Diversity scans $(\pi)$ for all chromosomes 
a

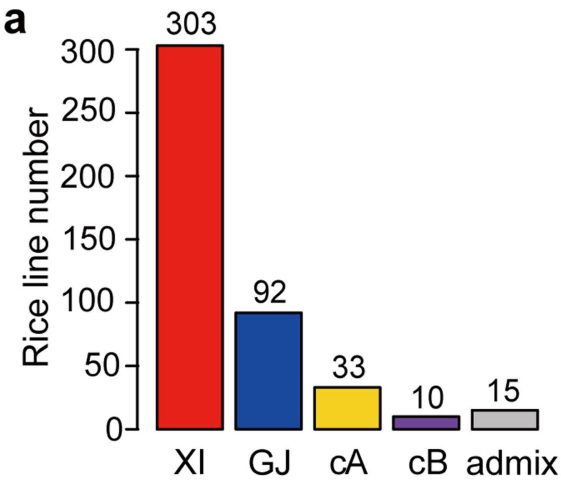

b

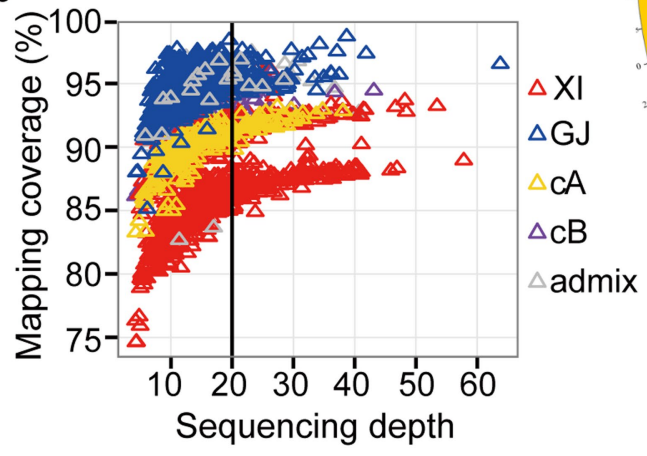

Extended Data Fig. 4 | Selection of high-depth accessions and summary of SVs. a, Number of accessions with sequencing depths $\geq 20 \times$ and mapping depth $\geq 15 \times$. b. Mapping coverage of the 3,010 rice genomes to the Nipponbare RefSeq as a function of sequence depth. c, Circular presentation of different types of structural variation detected in 453 highcoverage rice genomes when compared against the Nipponbare RefSeq. $\mathrm{Chr}$, outermost circle represents 12 rice chromosomes with marks in $\mathrm{Mb}$; Repeat, red heat map represents repeat content in 500-kb windows; DEL,

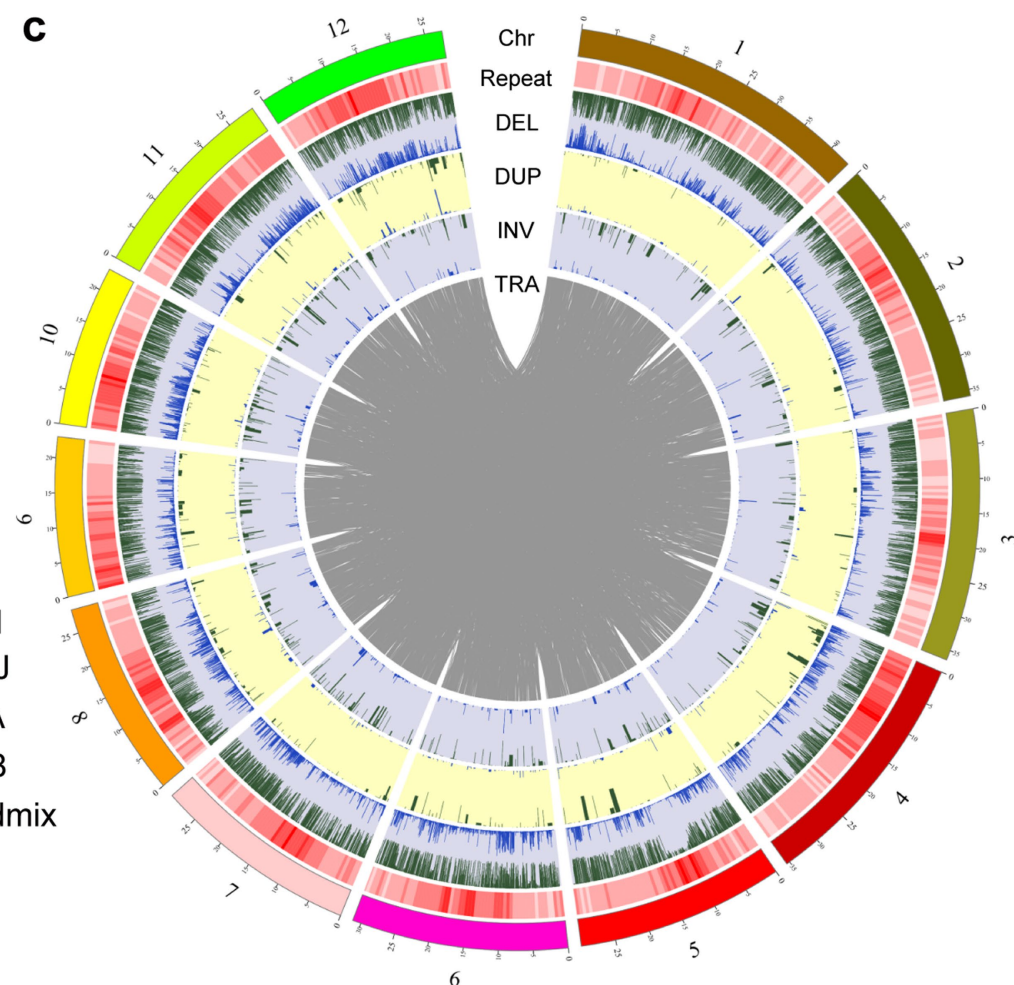

green/blue colour with inner/outer bars represents the average frequencies of deletions detected in XI and GJ; DUP, green/blue colour with inner/ outer bars represents the average frequencies of duplications detected in $\mathrm{XI}$ and GJ; INV, green/blue colour with inner/outer bars represents the average frequencies of inversions detected in XI and GJ; TRA, grey colour represents translocations across each genome with an average frequency $>0.3$ in either XI or GJ. 

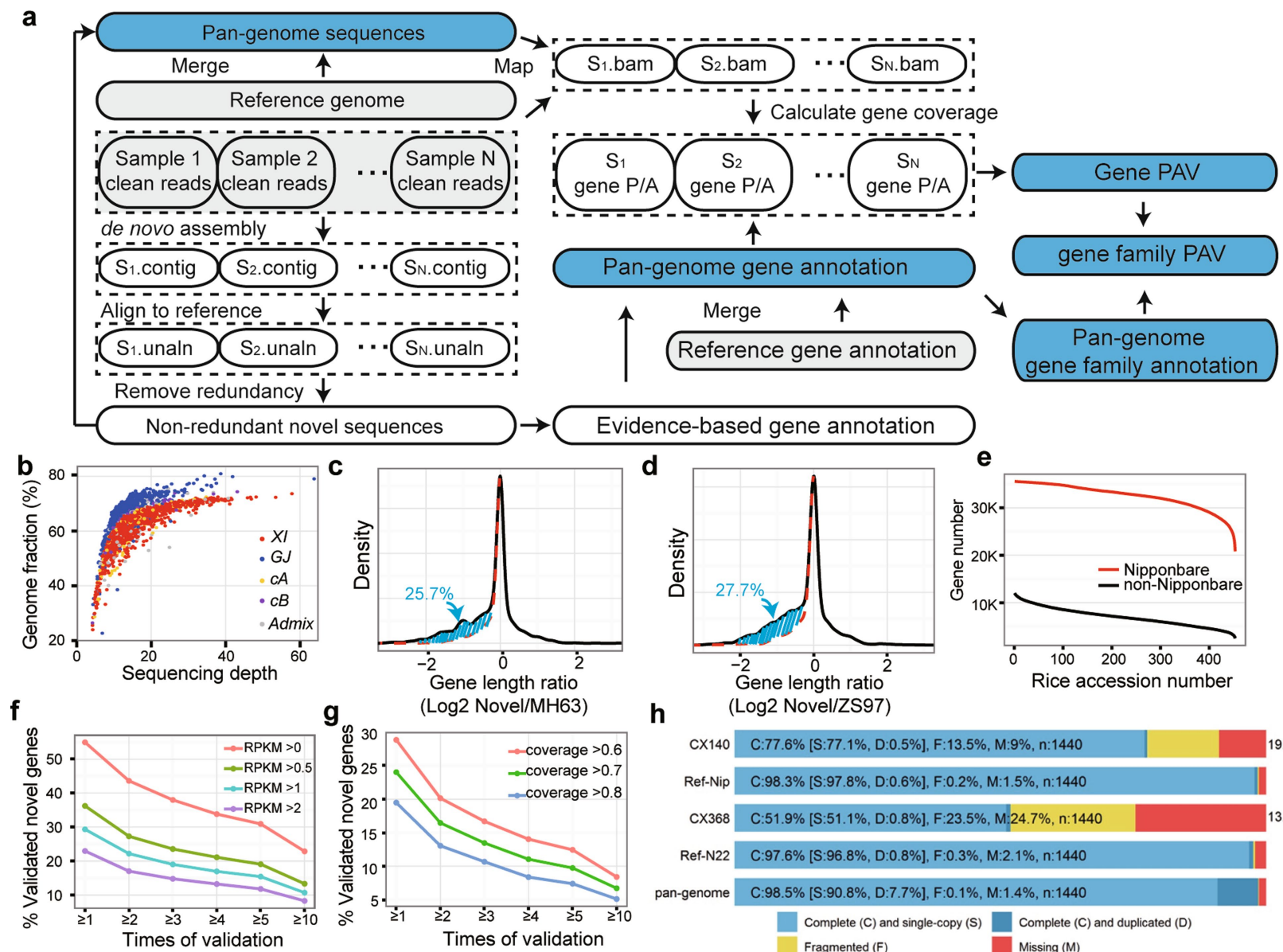

Evidence-based gene annotation

d
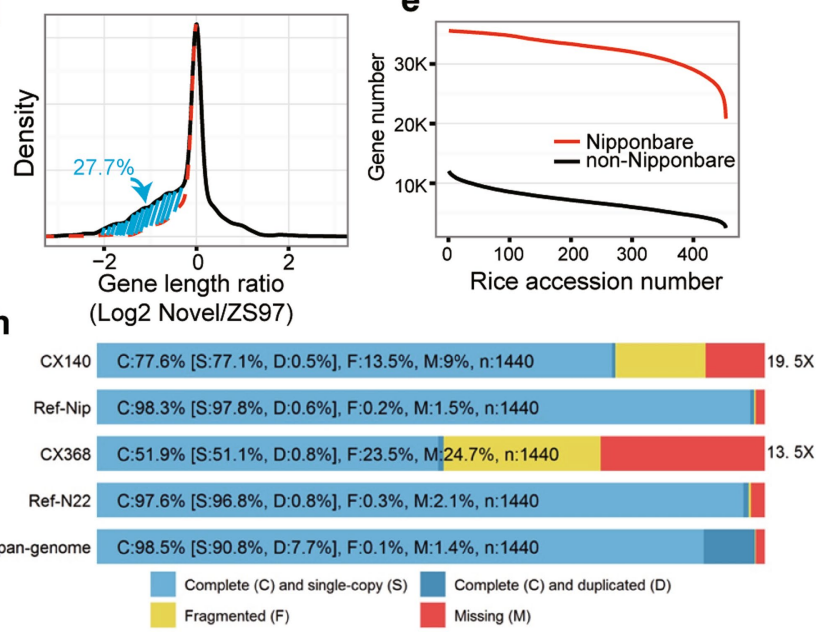

novel gene is longer and a ratio $<0$ means the novel gene is shorter. The dashed red lines show the symmetric distributions of the $>0$ part and the blue regions show the gene proportion with shorter lengths. e-g, Genomic (e) and transcriptomic (f, $\mathbf{g})$ validation of novel genes. e, Validation based on genomic sequencing data, in which numbers of the Nipponbare RefSeq and non-Nipponbare RefSeq genes identified (>95\% CDS coverage and $>85 \%$ gene-body coverage) are shown against the numbers of supporting rice accessions in the 453 rice lines; $\mathbf{f}, \mathbf{g}$, Validation based on the mapping rates of the publicly available RNA sequencing data of rice, including gene expression (f) and coverage of the coding sequence $(\mathbf{g})$. $\mathbf{h}, \mathrm{BUSCO}$ evaluation for 1,440 highly conserved genes; CX140, the assembly of Illumina sequencing data of Nipponbare accessions; Ref-Nip, Nipponbare RefSeq. CX368, the assembly of Illumina sequencing data of accession N 22; Ref-N22, assembly of N 22 PacBio sequencing data. 
a

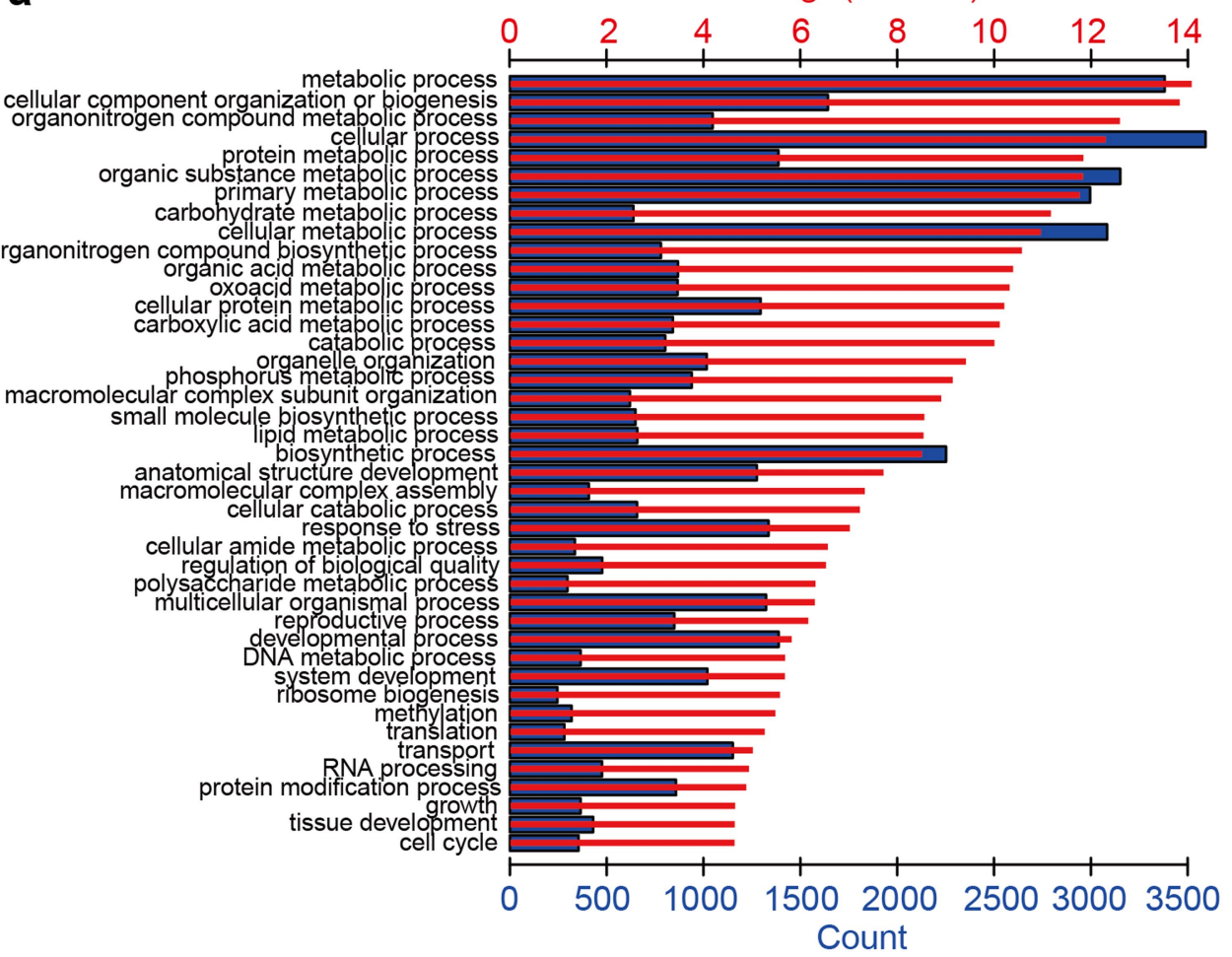

b

- $\log _{10}(P$-value $)$

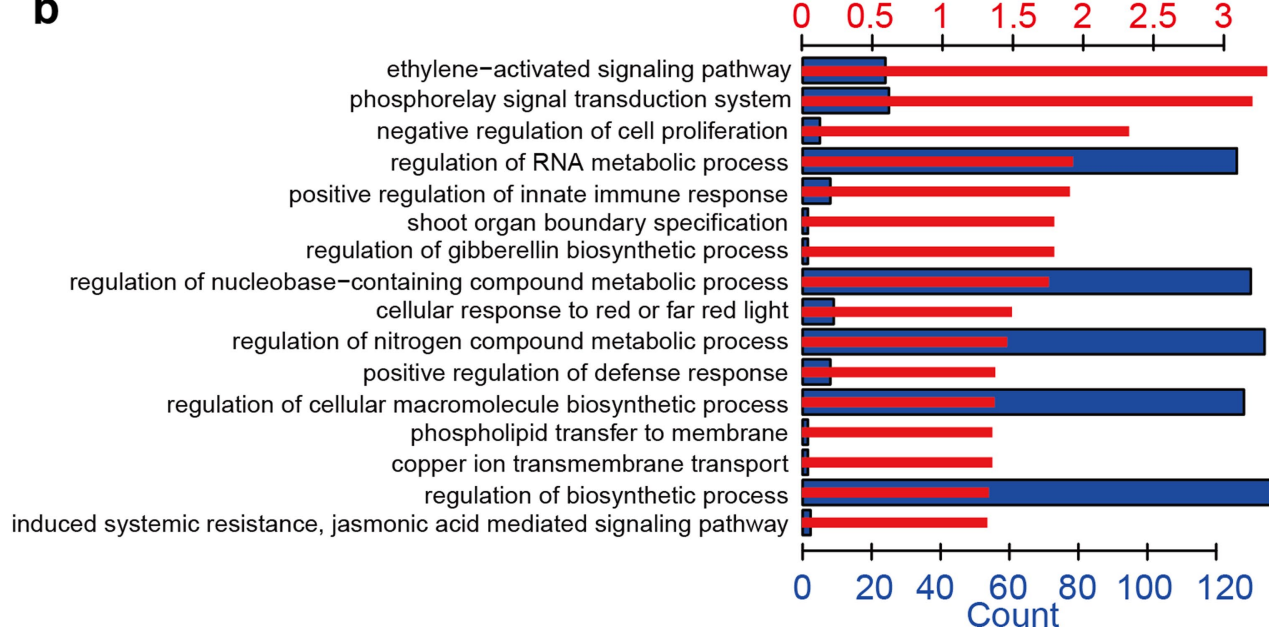

Extended Data Fig. 6 | Representative enriched biological processes of core and distributed gene families. a, b, Representative enriched biological processes of core (a) and distributed gene families $(\mathbf{b})$ are shown, with all terms sorted by their enriched $P$ values (red bars).
One-sided hypergeometric test built in the GOstats $\mathrm{R}$ package was used to calculate the $P$ value of each GO term. The numbers of gene families involved in each GO term are shown in blue. 
a

b

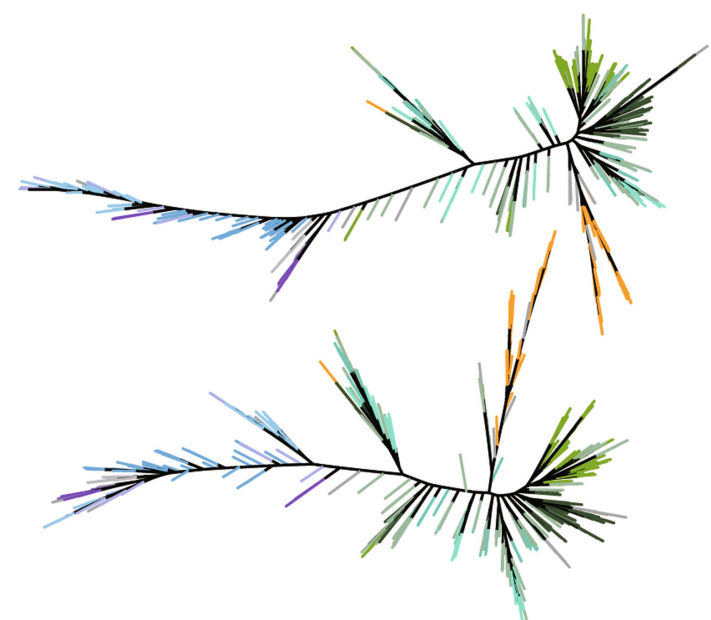

$\square \mathrm{XI}-1 \mathrm{~A} \square \mathrm{XI}-1 \mathrm{~B} \square \mathrm{XI}-2 \square \mathrm{XI}-3 \square \mathrm{XI}-\mathrm{adm}$ $\square$ GJ-trp $\square$ GJ-sbtrp $\square$ GJ-tmp $\square$ GJ-adm $\square \mathrm{cA} \quad \square \mathrm{cB} \quad \square$ admix

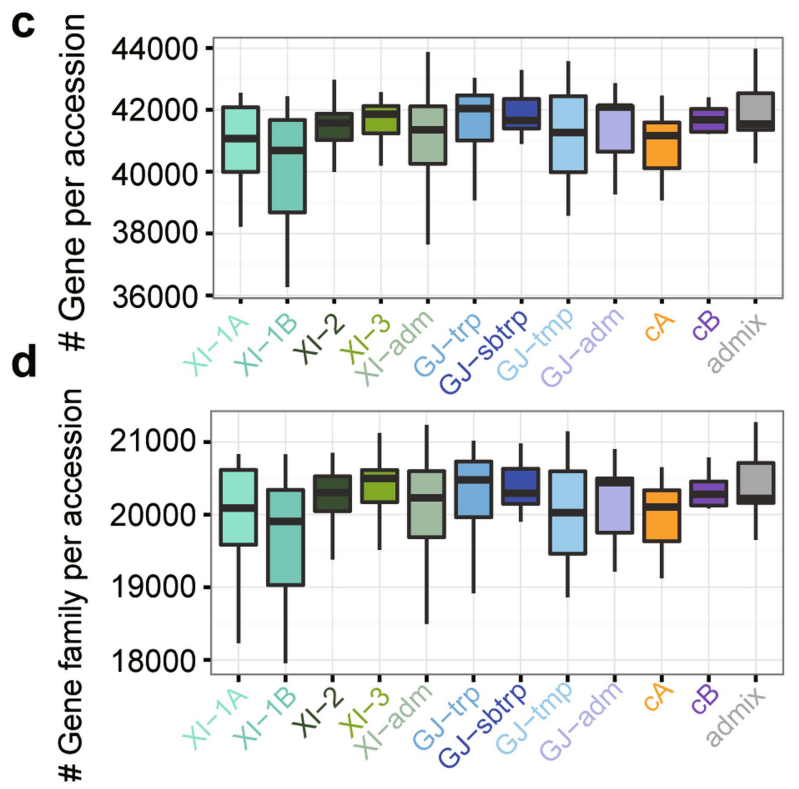

Extended Data Fig. 7 | Characterization of gene or gene family presence or absence variations. a, b, Phylogenetic trees of the 453 rice accessions constructed on the basis of the presence or absence of the distributed genes (a) and gene families (b); both of which classified the 453 accessions into two major groups (XI and GJ), with each being further divided into several subpopulations that are tagged with different colours representing their classifications based on the SNP. c, d, Gene (c) or gene family (d) numbers per accession in different subpopulations; gene or gene family numbers were significantly different among XI subpopulations (Kruskal-Wallis tests, $P$ value $=9.8 \times 10^{-8}($ gene $)$ or $1.0 \times 10^{-6}($ gene family $\left.)\right)$. Box plots e
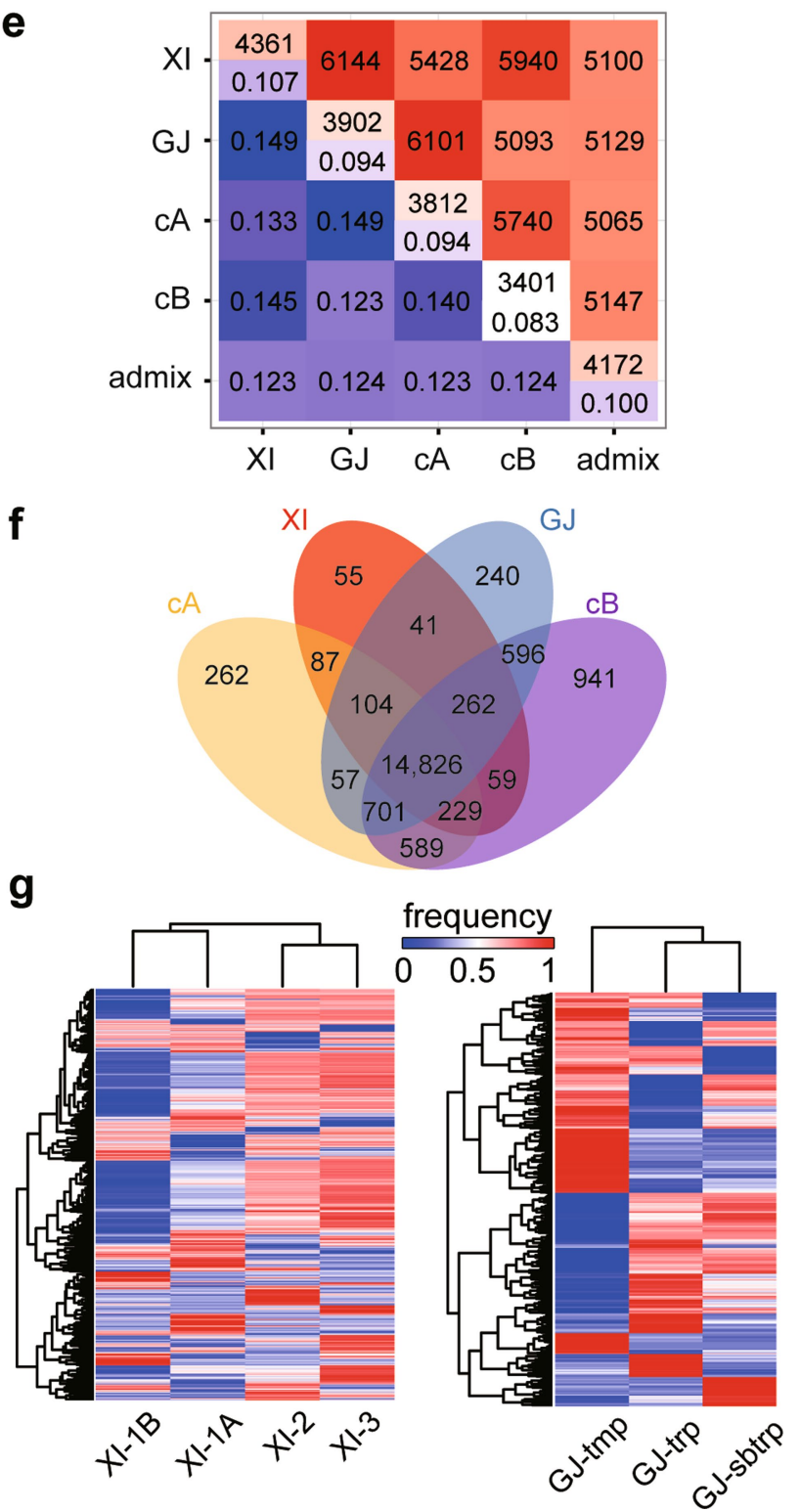

show the median, box edges represent the first and third quartiles and the whiskers extend to $1.5 \times$ interquartile range. $\mathbf{e}$, The average number of genes that are different between two accessions in which all combinations of the 453 accessions were considered, and the proportions were calculated as the number of such differentiating genes adjusted by the gene numbers held in common by the two genome types. $f$, Venn diagram of the numbers of the core + candidate core gene families among the major groups of O. sativa. g, Cluster analysis of 4,270 XI-subpopulation-unbalanced gene families and 1,384 GJ-subpopulation-unbalanced gene families. 
a

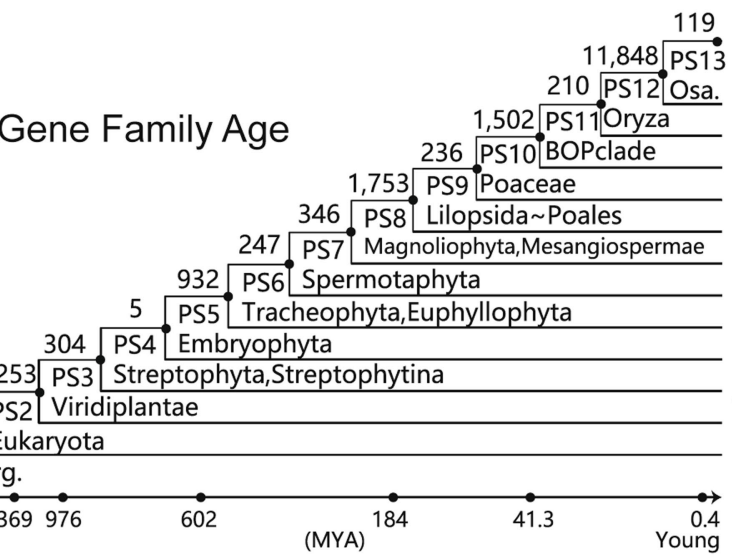

C

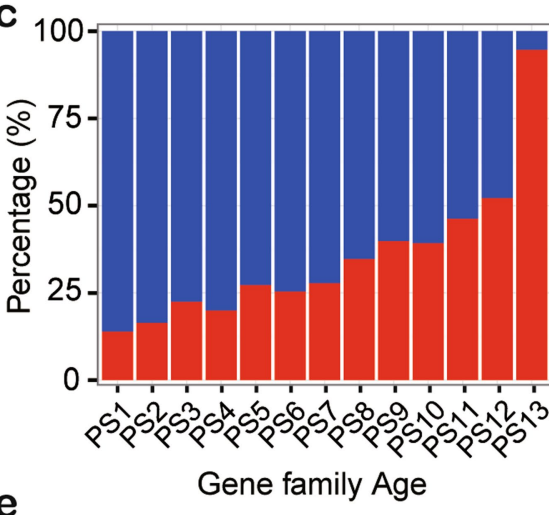

e

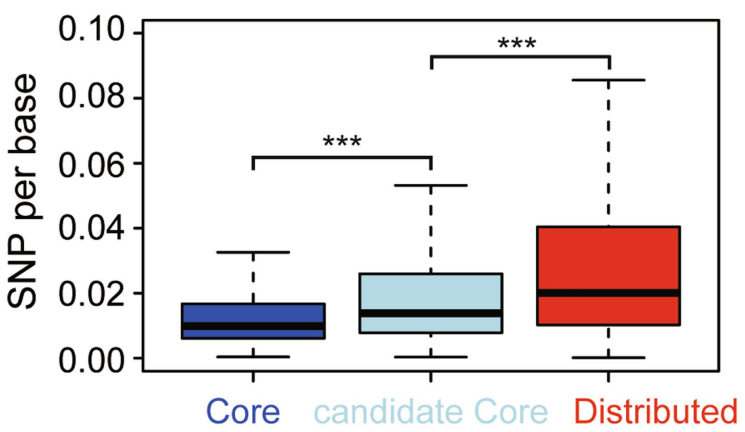

Extended Data Fig. 8 Evolution of the pan-genome of O.sativa.

$\mathbf{a}, \mathbf{b}$, The numbers of gene families (a) and genes (b) that emerged at different evolutionary times, from PS1 (single-cell organisms) to PS13 (O. sativa). c, The age distribution of the core and distributed gene families. d, Coding sequence length distribution for Nipponbare RefSeq genes with different ages. e, $\mathbf{f}$, SNP variation of the core and distributed genes against the Nipponbare RefSeq. e, The density of SNPs in the coding region of core and distributed genes in the 3,010 rice lines; SNP density in core genes is lower than that in candidate core genes (two-sided b

168

$14,0 7 2 \longdiv { \text { PS1 } }$

385 PS12 Osa.

Gene Age

2,817 PS11 Oryza

473 PS10 BOPclade

3,861 PS9 Poaceae

757 PS8 Lilopsida Poales

659 PS7 Magnoliophyta,Mesangiospermae 2,599 PS6 Spermotaphyta

11 PS5 Tracheophyta,Euphyllophyta

667 PS4 Embryophyta

5,763 PS3 Streptophyta,Streptophytina

15,866 PS2 Viridiplantae

PS1 Eukaryota

Cell.org.

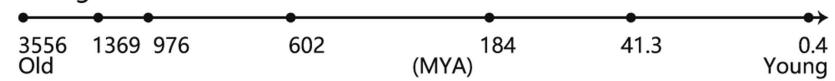

d

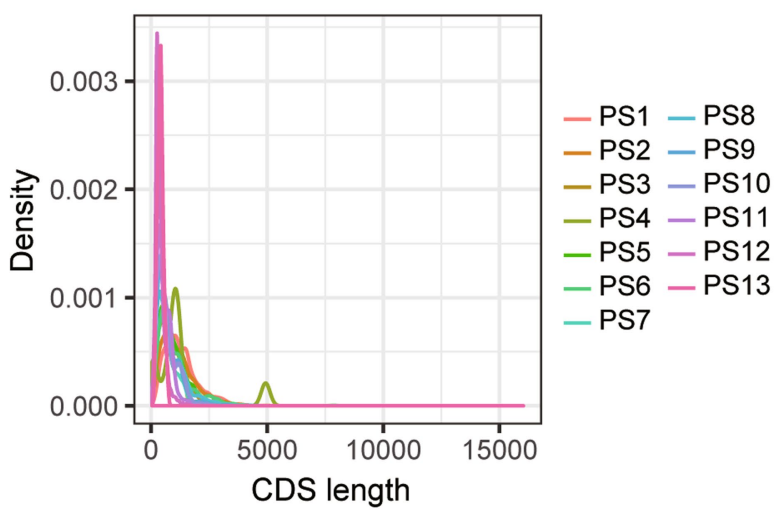

f

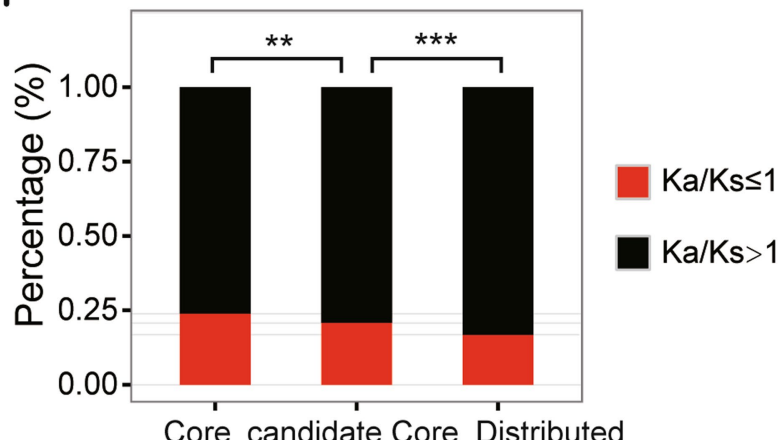

Wilcoxon test) and SNP density in candidate core genes is lower than that in distributed genes (two-sided Wilcoxon test). Box plots show the median, box edges represent the first and third quartiles, and the whiskers extend to $1.5 \times$ interquartile range. f, $K_{\mathrm{a}} / K_{\mathrm{s}}$ of the core and distributed genes. After removing genes with no synonymous SNPs, there are 3,144 core, 455 candidate core and 800 distributed genes with $K_{\mathrm{a}} / K_{\mathrm{s}}>1$ and 10,005 core, 1,727 candidate core and 3,957 distributed genes with $K_{\mathrm{a}} / K_{\mathrm{s}}$ $<1$. Two sided $\chi$-square tests were used to determine the difference of the proportions. $* * P<0.01, * * * P<0.001$. 
a

a

Rc

el,

deletion presence/absence

GJ

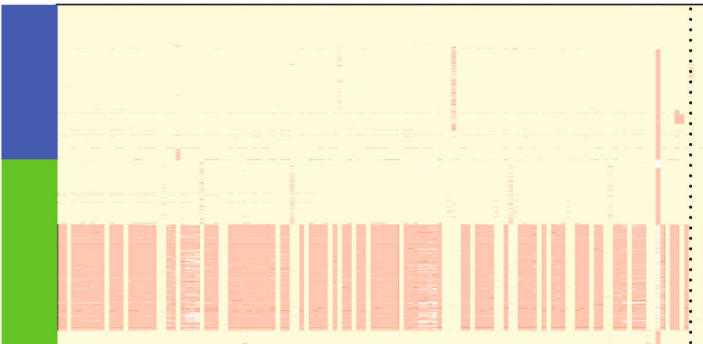

$c \mathrm{~B}$

admix

b

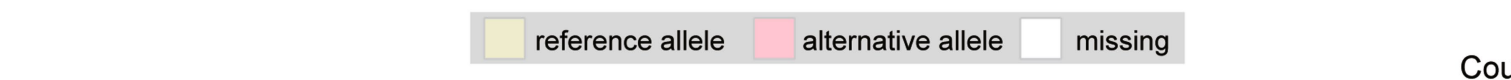

Introgressed haplotypes
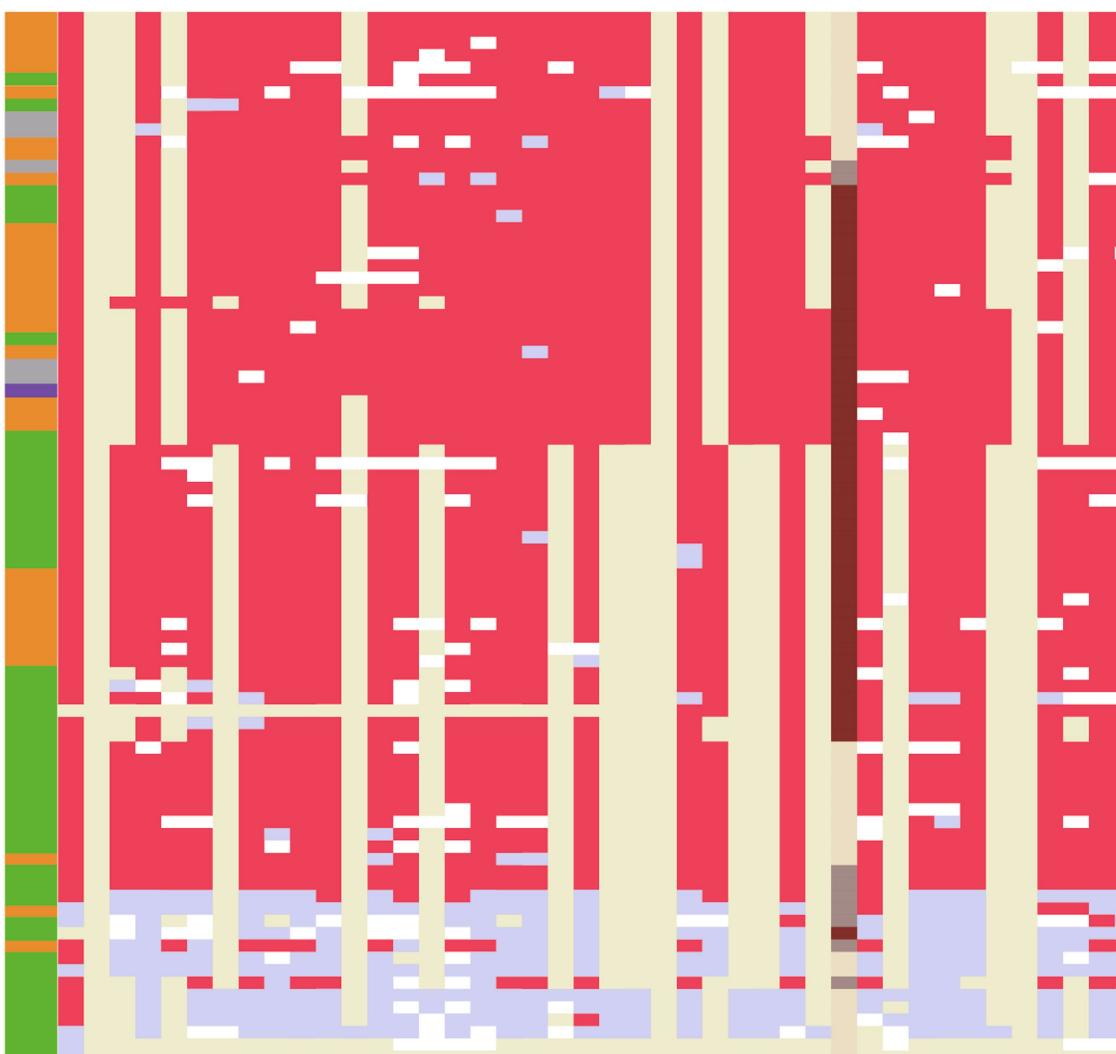

allele code

0

2 del het wt

Country

Bangladesh

China Indian Indonesia

Philippines Pakistan

Sri Lanka

Viet Nam other-Asia non-Asia

Extended Data Fig. 9 | Haplotypes around the red pericarp $(R c)$ gene. Rows correspond to samples and columns correspond to SNPs. The colour of the rectangle denotes the number of non-Nipponbare alleles in a genotype. a, Haplotypes of 3,010 samples in a region $\pm 25 \mathrm{~kb}$ around $R c$ show the presence of many distinctly non-GJ haplotypes that carry either the wild-type or domesticated allele at the causative deletion site b, Zoomed-in view of a subset of 90 samples and 53 SNPs within the $R c$ gene and $15-\mathrm{kb}$ downstream highlights the wide dispersal of non-GJ domesticated haplotypes. 


\section{RESEARCH ARTICLE}
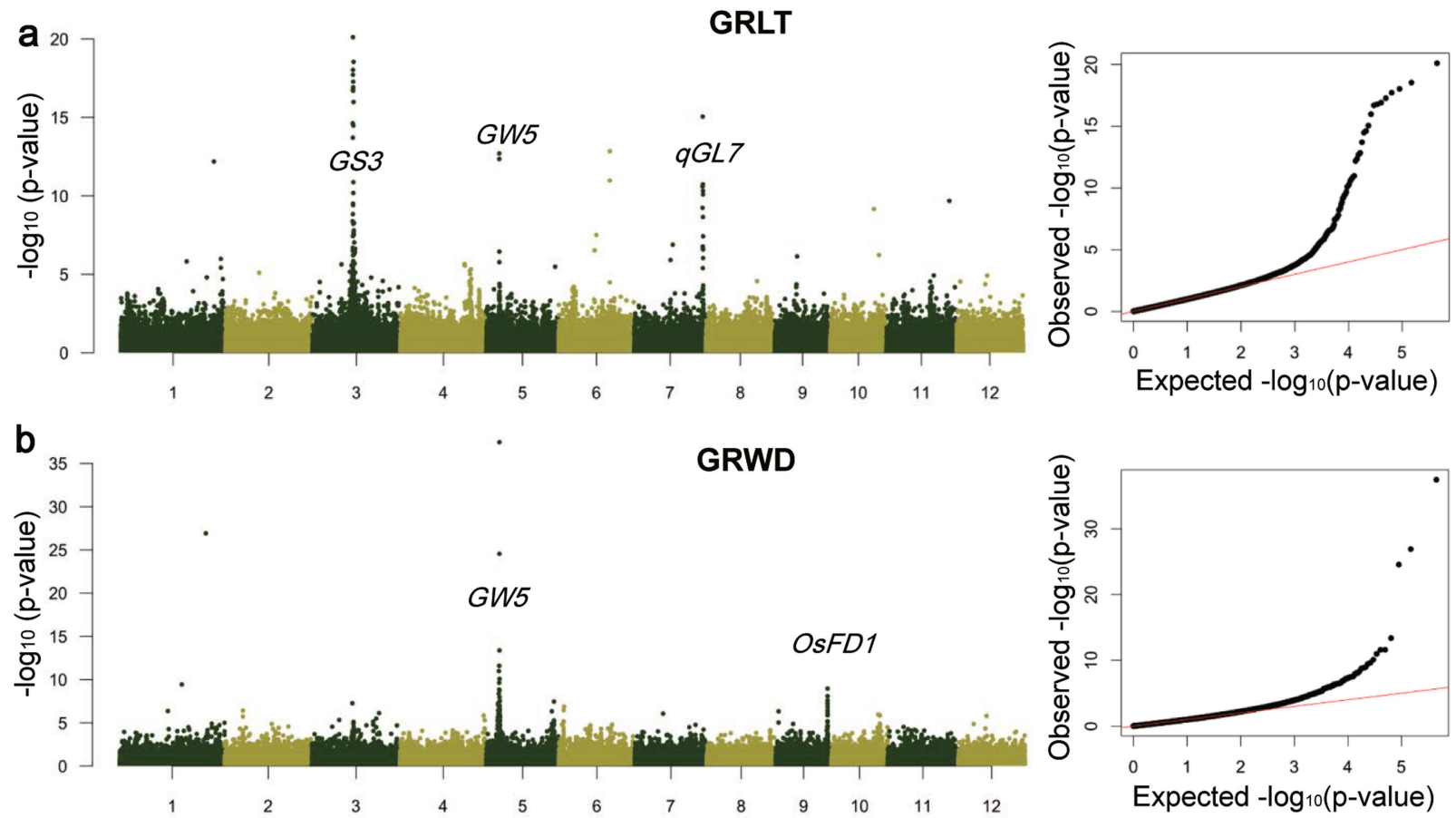

C

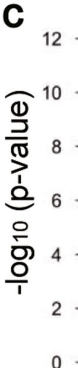

BBL C5

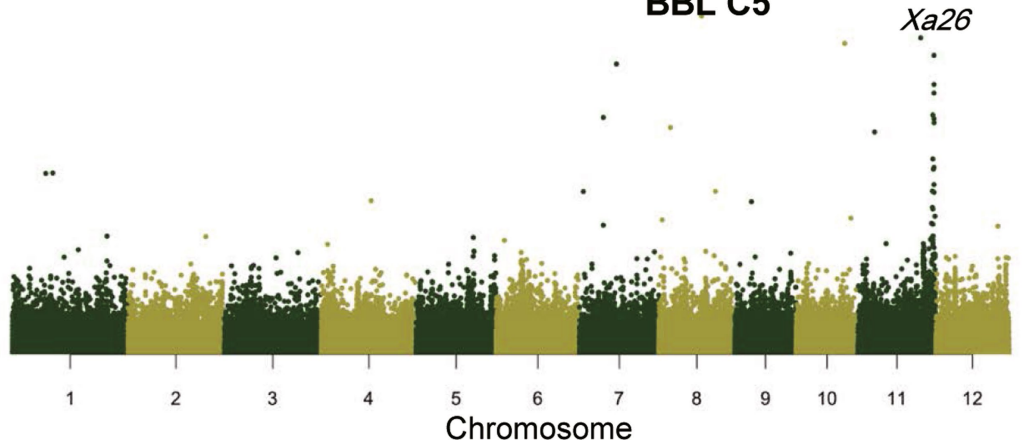

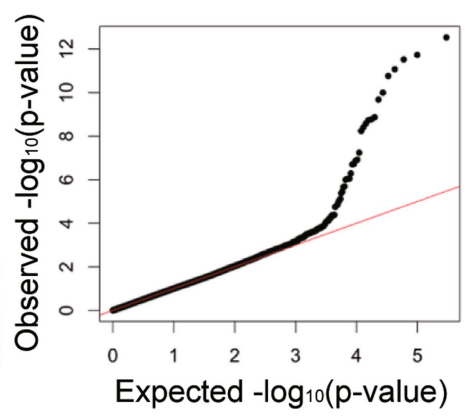

Extended Data Fig. 10 | Genome-wide association for grain length, grain width and bacterial blight isolate C5. a-c, GWAS for grain length (GRLT, $n=2,012)$ (a), grain width (GRWD, $n=2,012$ ) (b) and bacterial blight isolate C5 (BBL C5, $n=381$ ) (c). GWAS was performed using filtered and linkage disequilibrium-pruned SNPs for historical trait data on source accessions for grain length and grain width (223,743 SNPs) and for newly acquired lesion length data for bacterial blight isolate C5 (148,999 SNPs). Manhattan plots for linkage disequilibrium-pruned datasets are shown to the left and quartile-quartile plots for expected versus observed $-\log (P)$ values to the right. Major peaks are annotated for known gene loci. 
a

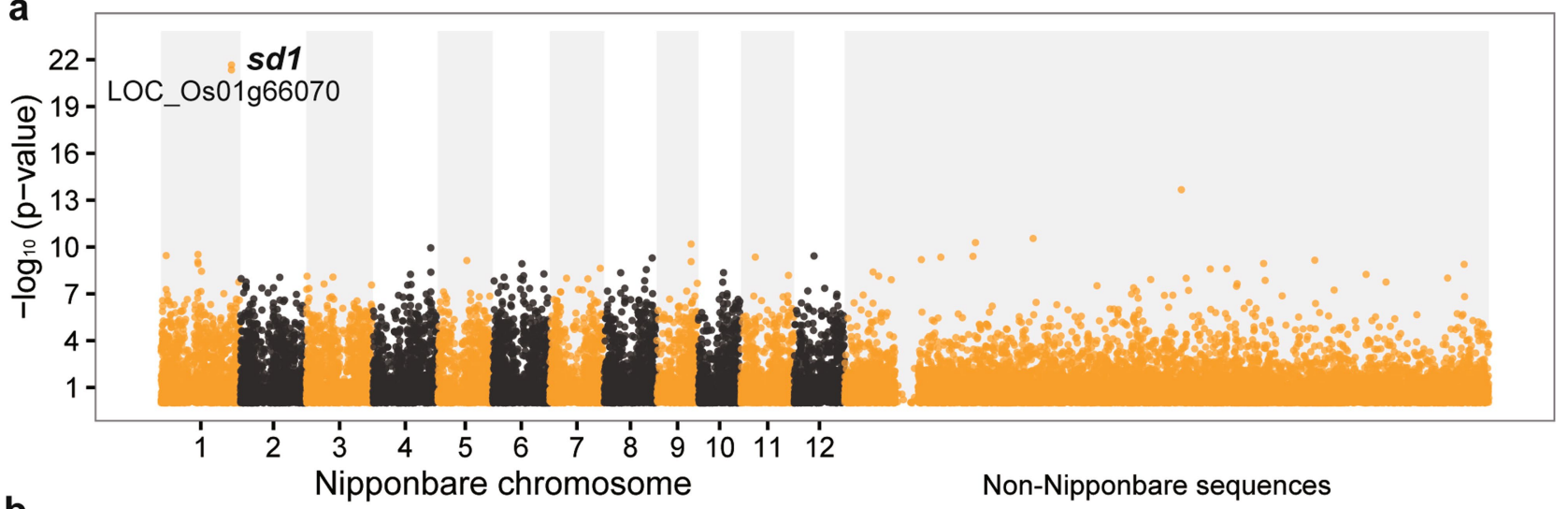

b

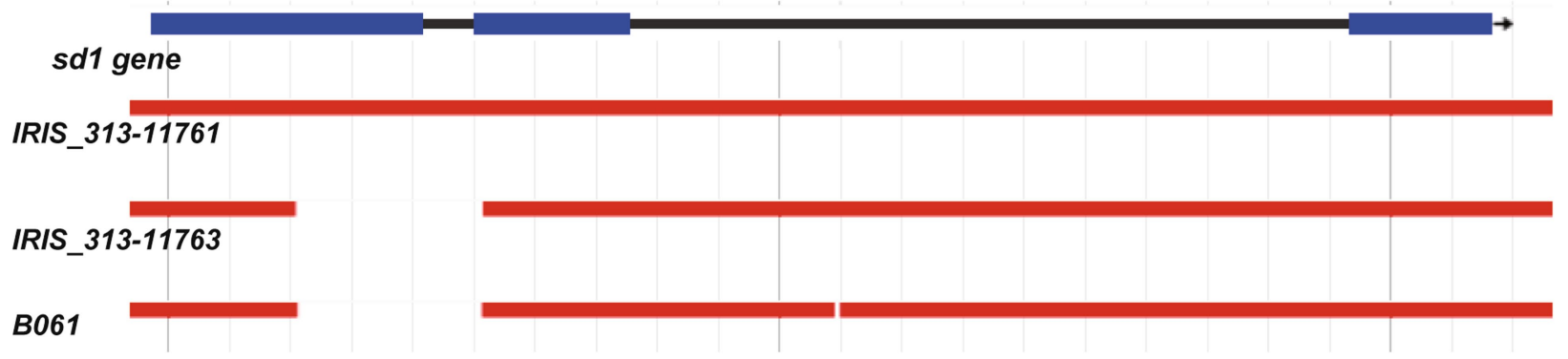

C

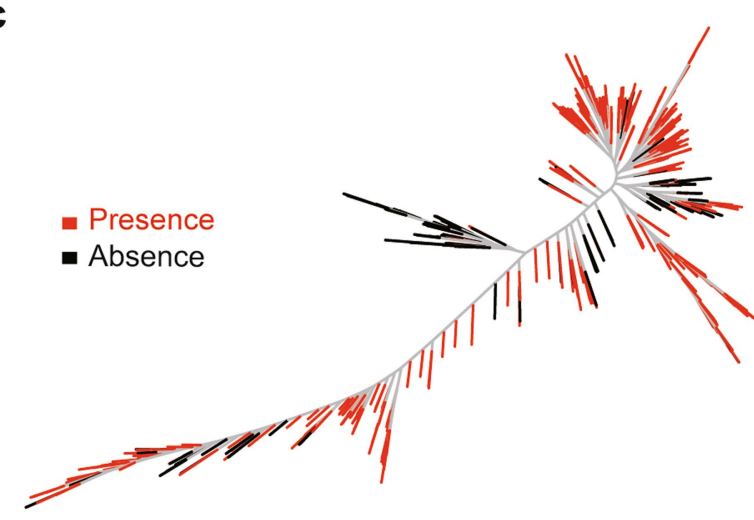

Extended Data Fig. $11 \mid s d 1$ gene and its correlation with plant height. a, The plot shows the correlation of the gene presence or absence variation with plant height $(n=323)$. The $P$ values were calculated with Spearman's correlation. b, Examples show that semi-dwarfism results

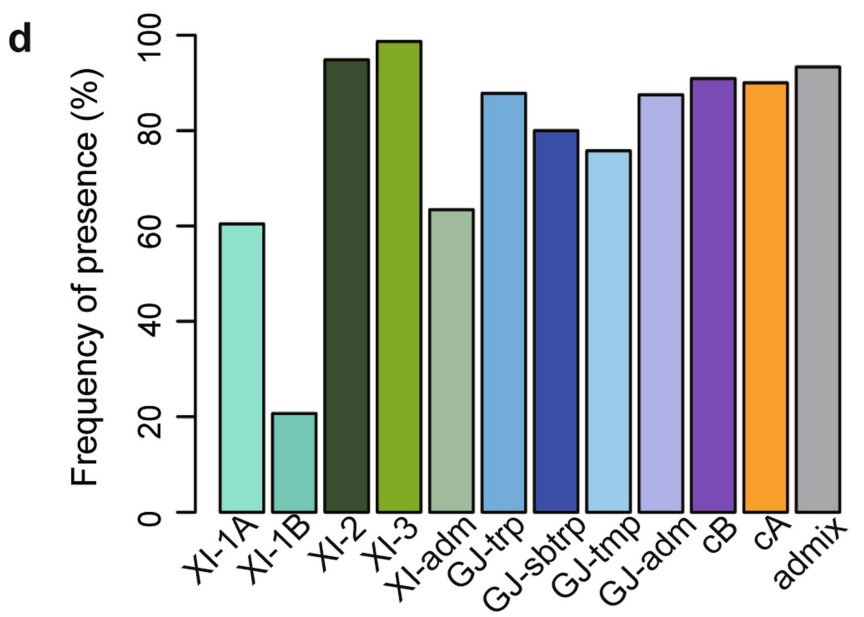

from an approximately 385 -bp deletion in the $s d 1$ locus. c, Distribution of the presence or absence of the $s d 1$ gene in the 453 rice accessions. $d, s d 1$ frequencies in rice subpopulations. 


\section{nature research}

Corresponding author(s):

Initial submission
Zhikang Li

Revised version

$\bigotimes$ Final submission

\section{Life Sciences Reporting Summary}

Nature Research wishes to improve the reproducibility of the work that we publish. This form is intended for publication with all accepted life science papers and provides structure for consistency and transparency in reporting. Every life science submission will use this form; some list items might not apply to an individual manuscript, but all fields must be completed for clarity.

For further information on the points included in this form, see Reporting Life Sciences Research. For further information on Nature Research policies, including our data availability policy, see Authors \& Referees and the Editorial Policy Checklist.

\section{- Experimental design}

1. Sample size

Describe how sample size was determined.

\section{Data exclusions}

Describe any data exclusions.

\section{Replication}

Describe whether the experimental findings were reliably reproduced.

\section{Randomization}

Describe how samples/organisms/participants were allocated into experimental groups.

5. Blinding

Describe whether the investigators were blinded to group allocation during data collection and/or analysis.
We sequenced 3,024 representative rice accessions from 89 countries all over the world, including 2,466 accessions from the International Rice Research Institute (IRRI), and 558 accessions from the Chinese Academy of Agricultural Sciences (CAAS). The 2,466 accessions contributed by IRRI represent a panel that was randomly selected from a core collection of 12,000 O. sativa accessions that was established by a semi-stratified selection scheme from more than 101,000 rice accessions. The 558 accessions contributed by CAAS included a mini-core collection of 246 accessions selected from a core collection of 932 accessions established in the same way from the 61,470 O. sativa accessions, plus 312 accessions selected based on their isozyme diversity, and used as parental lines in the international rice molecular breeding network.

The 453 accessions selected for SV and gene PAV analysis are randomly distributed and we demonstrated that they can represent the population structure.

14 accessions were excluded due to either extremely low sequencing depth or large proportion of contaminants. We described each accession in detail in the Supplementary Notes.

Replication of this study's findings was not attempted.

The 453 accessions with sequencing depth $>20 x$ and mapping depth $>15 x$ were selected for SV and gene PAV analysis are purely based on sequencing depth and they are randomly distributed. We demonstrated that they can represent the population structure.

No blinding needed. All rice sequencing data are processed equally. 
6. Statistical parameters

For all figures and tables that use statistical methods, confirm that the following items are present in relevant figure legends (or in the Methods section if additional space is needed).

$\mathrm{n} / \mathrm{a} \mid$ Confirmed

$\bigotimes$ The exact sample size $(n)$ for each experimental group/condition, given as a discrete number and unit of measurement (animals, litters, cultures, etc.)

$\square$ A description of how samples were collected, noting whether measurements were taken from distinct samples or whether the same

sample was measured repeatedly

$\bigotimes$ A statement indicating how many times each experiment was replicated

The statistical test(s) used and whether they are one- or two-sided (note: only common tests should be described solely by name; more

complex techniques should be described in the Methods section)

$\bigotimes$ A description of any assumptions or corrections, such as an adjustment for multiple comparisons

$\bigotimes$ The test results (e.g. $P$ values) given as exact values whenever possible and with confidence intervals noted

$\bigotimes$ A clear description of statistics including central tendency (e.g. median, mean) and variation (e.g. standard deviation, interquartile range)

$\square$ Clearly defined error bars

See the web collection on statistics for biologists for further resources and guidance.

- Software

Policy information about availability of computer code

7. Software

Describe the software used to analyze the data in this study.

FastQC v0.11.2; BWA v0.7.10; samtools v1.0; Picatd tools release 1.119; GATK release 3.2-2; SnpEff; MUMmer v3; EMMAX algorithm implemented in SNP \& Variation Suite v8.4.0; R 'qqman' package v.0.1.3; PLINK v1.90b1g; PHYLIP v3.695; 'cmdscale' function in R v.3.3.1; ADMIXTURE v.1.3; CLUMPP v.1.1.2; R v.3.3.1, with custom scripts at https://github.com/dchebotarov/3k-SNP-paper; tailed novoBreak, avaliable at https://sourceforge.net/projects/novobreak/? source=navbar ; SOAPdenovo2; GapCloser v1.1.2; Qualimap v2.026; BUSCO, with Augustus 3.2.3, hmmer 3.1b; FALCON; Canu; Quiver; Pilon; Genome Puzzle Master; bamUtil v1.0.12; CD-HIT v4.6; BEDtools v2.17.0; MAKER 2, with SNAP, AUGUSTUS; HISAT2 version 2.0.1-beta; OrthoMCL v2.0; kmer_count; BLAST V2.2.28+; megaBLAST We described the usage of each software together with the command line in detail in the Supplementary Notes.

For manuscripts utilizing custom algorithms or software that are central to the paper but not yet described in the published literature, software must be made available to editors and reviewers upon request. We strongly encourage code deposition in a community repository (e.g. GitHub). Nature Methods guidance for providing algorithms and software for publication provides further information on this topic.

\section{- Materials and reagents}

Policy information about availability of materials

8. Materials availability

Indicate whether there are restrictions on availability of unique materials or if these materials are only available for distribution by a for-profit company.

\section{Antibodies}

Describe the antibodies used and how they were validated for use in the system under study (i.e. assay and species).
No unique materials were used.

No antibodies were used. 
10. Eukaryotic cell lines

a. State the source of each eukaryotic cell line used.

No eukaryotic cell lines were used.

b. Describe the method of cell line authentication used.

No eukaryotic cell lines were used.

c. Report whether the cell lines were tested for mycoplasma contamination.

d. If any of the cell lines used are listed in the database of commonly misidentified cell lines maintained by ICLAC, provide a scientific rationale for their use.

No eukaryotic cell lines were used.

No eukaryotic cell lines were used.

- Animals and human research participants

Policy information about studies involving animals; when reporting animal research, follow the ARRIVE guidelines

11. Description of research animals

Provide details on animals and/or animal-derived

No animals were used.

materials used in the study.

Policy information about studies involving human research participants

12. Description of human research participants

Describe the covariate-relevant population

characteristics of the human research participants.

The study didn't involve human participants. 